\title{
PRICING OF FORWARDS AND OPTIONS IN A MULTIVARIATE NON-GAUSSIAN STOCHASTIC VOLATILITY MODEL FOR ENERGY MARKETS
}

\author{
F. E. BENTH, ${ }^{* * *}$ University of Oslo \\ L. VOS, * University of Oslo and University of Agder
}

\begin{abstract}
In Benth and Vos (2013) we introduced a multivariate spot price model with stochastic volatility for energy markets which captures characteristic features, such as price spikes, mean reversion, stochastic volatility, and inverse leverage effect as well as dependencies between commodities. In this paper we derive the forward price dynamics based on our multivariate spot price model, providing a very flexible structure for the forward curves, including contango, backwardation, and hump shape. Moreover, a Fourier transformbased method to price options on the forward is described.
\end{abstract}

Keywords: Energy market; forward pricing; spread option; subordinator; stochastic volatility

2010 Mathematics Subject Classification: Primary 60G10

Secondary 91G20; 15A04; 60G51; 60H30

\section{Introduction}

During the last decades the energy markets have been liberalized worldwide, resulting in market places for commodities such as electricity, gas, and coal. There are several markets for each of these commodities, geographically spread over the continents. For example, in Europe we have markets for power in the UK, Germany, France, and the Nordic countries, to mention a few. There are transmission lines which interconnect these markets for electricity. Furthermore, since coal and gas are used to a large extent as fuels for power production, the prices for these commodities naturally impact the power prices. These markets become more and more integrated, both within one commodity, but also across the commodities. For this reason, there is an increasing interest in studying multivariate models for energy markets, including crosscommodity models (such as, for example, gas, coal, and electricity) or multivariate models for the same commodity traded in different, but integrated markets (such as, for example, the power markets in the Nordic countries and Germany).

In Benth and Vos [6] we proposed a stochastic dynamic for cross-commodity spot price modeling, generalizing the univariate dynamics studied by Benth [5]. The model is flexible enough to capture spikes and mean reversion. Moreover, it includes the possibility to model inverse leverage and stochastic volatility. The proposed dynamics can model co- and independent jump behavior (spikes) in cross-commodity markets, and is analytically tractable. We apply the multivariate extension of the stochastic volatility model of Barndorff-Nielsen and

Received 22 November 2011; revision received 7 August 2012.

* Postal address: Centre of Mathematics for Applications, University of Oslo, PO Box 1053, Blindern, N-0316 Oslo, Norway.

** Email address: fredb@math.uio.no 
Shephard [2], analyzed in detail by Pigorsch and Stelzer [16]. The mean-reverting features of our spot model require a significant extension of their analysis.

In this paper we derive the forward dynamics using a noarbitrage pricing. Despite the rather general nature of our spot model, the dynamics of the forward prices are analytically computable. It turns out that the implied forward curves can be in contango and backwardation, as well as having humps. As has been pointed out by Geman [11], hump-shaped forward curves have been observed in, for instance, the oil market. Due to the flexibility of the multivariate model, even an oscillation of the forward price curve can be achieved. As an implication of the stationarity properties of the spot model, the forward prices in the long end of the forward curve (far until maturity) will move deterministically. The Samuelson effect can be identified in the forward dynamics as well.

By using Fourier methods, options on spreads between different forward contracts can be represented as integrals which can be computed efficiently. Spread options are traded for in various energy markets, mostly over-the-counter. However, such options are also used in valuation of new power plant projects and the construction of interconnecting pipelines between different markets. In fact, the construction of a new pipeline connecting two markets can be viewed as a long-term spread option. On the other hand, the value of a gas-fired power plant can be represented as a spread between electricity and gas (so-called spark spread).

The paper is organized as follows. In Section 2 we recall the spot model proposed in Benth and Vos [6]. Next, in Section 3, the implied multivariate forward dynamics are derived and properties of the forward curve are analyzed. Methods based on the Fourier transform are applied to cross-commodity option pricing in Section 4, with special attention paid to spread options. Finally, in Section 5 we conclude.

\section{A cross-commodity energy spot price model with stochastic volatility}

In this section we briefly recall the main aspects of the spot model with stochastic volatility for cross-commodity energy markets introduced in Benth and Vos [6]. We suppose that we are given a complete filtered probability space $(\Omega, \mathcal{F}, \mathbb{P})$ equipped with the filtration $\left\{\mathcal{F}_{t}\right\}_{t \geq 0}$ satisfying the usual conditions (see, e.g. [17]).

Assume that $m \leq n \in \mathbb{N}$, and, for $d \in \mathbb{N}$, consider a $d$-dimensional spot price dynamic as a combination of a seasonality function $\Lambda$, stochastic processes $\left\{Y_{i}\right\}_{i=1}^{m}$ modeling spikes, and a stochastic process $X$ modeling the 'normal' variations of the price evolution. Here, the seasonality and the stochastic processes $X$ and $\left\{Y_{i}\right\}_{i=1}^{m}$ are all $d$-dimensional. More precisely, we define the spot price dynamics of $d$ energy commodities as follows:

$$
S(t)=\Lambda(t) \cdot \exp \left(X(t)+\sum_{i=1}^{m} Y_{i}(t)\right) .
$$

Here the dot denotes pointwise multiplication, and the seasonality $\Lambda$ is supposed to be a deterministic measurable function. The stochastic processes $\left\{Y_{i}\right\}_{i=1}^{m}$ are $d$-dimensional Ornstein-Uhlenbeck processes driven by vector-valued subordinators $\left\{L_{i}\right\}_{i=1}^{m}$, that is, Lévy processes which are increasing in each of their coordinates (see [4]):

$$
\mathrm{d} Y_{i}(t)=\left(\mu_{i}+B_{i} Y_{i}(t)\right) \mathrm{d} t+\eta_{i} \mathrm{~d} L_{i}(t) .
$$

Here $\left\{\mu_{i}\right\}_{i=1}^{m}$ are vectors in $\mathbb{R}^{d}$. Furthermore, the $\left\{B_{i}\right\}_{i=1}^{m}$ are elements of $\mathrm{GL}_{d}(\mathbb{R})$, the group of $d \times d$ matrices which are invertible, while the $\left\{\eta_{i}\right\}_{i=1}^{m}$ are elements of $M_{d}(\mathbb{R})$, the space of 
real $d \times d$ matrices. The entries of $\eta_{i}$ do not necessarily have to be positive, so although the $L_{i}$ are subordinators, the process $Y_{i}$ may exhibit negative jumps. In electricity markets, say, negative spikes are observed.

The 'normal variations' process $X$ is an extension of the Barndorff-Nielsen and Shephard [2] stochastic volatility (BNS SV) model into the multidimensional Ornstein-Uhlenbeck setting. The stochastic process $X$ is defined by the stochastic differential equation

$$
\mathrm{d} X(t)=A X(t) \mathrm{d} t+\Sigma(t)^{1 / 2} \mathrm{~d} W(t)
$$

where $A$ is a matrix in $\mathrm{GL}_{d}(\mathbb{R})$ and $W$ is a standard $d$-dimensional Brownian motion in $\mathbb{R}^{d}$. The square of the volatility $\Sigma(t)$ is chosen to be a matrix-valued stochastic process. More precisely, the stochastic volatility $\Sigma(t)$ is a superposition of the positive-definite matrix-valued Ornstein-Uhlenbeck processes as introduced in [3], i.e.

$$
\Sigma(t)=\sum_{j=1}^{n} \omega_{j} Z_{j}(t)
$$

with

$$
\mathrm{d} Z_{j}(t)=\left(C_{j} Z_{j}(t)+Z_{j}(t) C_{j}^{\top}\right) \mathrm{d} t+\mathrm{d} \tilde{L}_{j}(t),
$$

where the $\omega_{j}$ s are positive weights summing up to 1 . Moreover, for $j=1, \ldots, n, C_{j} \in \mathrm{GL}_{d}(\mathbb{R})$ and $\widetilde{L}_{j}$ are independent matrix-valued subordinators, that is, independent increment processes with values in $\mathbb{S}_{d}^{+}$, the positive-definite cone of symmetric $d \times d$ matrices. Naturally, the $\widetilde{L}_{j}$ are independent of $W$ for $j=1, \ldots, n$, and we suppose for convenience that the subordinators are driftless. In order to have the Itô integrals in (2) well defined, we suppose that

$$
\mathbb{P}\left(\int_{0}^{\tilde{T}} \operatorname{tr}(\Sigma(t)) \mathrm{d} t<\infty\right)=1
$$

Here, $\widetilde{T}<\infty$ is some finite-horizon time for our energy markets, and tr is the trace operator on matrices. We assume that the eigenvalues of $C_{j}$ have negative real parts, a necessary condition for ensuring stationarity of the $Z_{j}$ s. We denote by $v_{\tilde{L}_{j}}$ the Lévy measure of $\widetilde{L}_{j}, j=1, \ldots, n$.

The processes $X$ and $Y_{i}$ are Ornstein-Uhlenbeck processes. Applying the multidimensional Itô formula (see [14]) yields the following explicit dynamics: for $0 \leq s \leq t$,

$$
\begin{aligned}
& X(t)=\mathrm{e}^{A(t-s)} X(s)+\int_{s}^{t} \mathrm{e}^{A(t-u)} \Sigma(u)^{1 / 2} \mathrm{~d} W(u), \\
& Y_{i}(t)=\mathrm{e}^{B_{i}(t-s)} Y_{i}(s)+B_{i}^{-1}\left(I-\mathrm{e}^{B_{i}(t-s)}\right) \mu_{i}+\int_{s}^{t} \mathrm{e}^{B_{i}(t-u)} \eta_{i} \mathrm{~d} L_{i}(u)
\end{aligned}
$$

for $i=1, \ldots, m$. The matrix exponentials are defined as usual as $\mathrm{e}^{A}:=I+\sum_{i=1}^{\infty} A^{n} / n !$.

According to Barndorff-Nielsen and Stelzer [3, Section 4], the solution of $Z_{j}(t), j=$ $1, \ldots, n$, is given by

$$
Z_{j}(t)=\mathrm{e}^{C_{j}(t-s)} Z_{j}(s) \mathrm{e}^{C_{j}^{\top}(t-s)}+\int_{s}^{t} \mathrm{e}^{C_{j}(t-u)} \mathrm{d} \widetilde{L}_{j}(u) \mathrm{e}^{C_{j}^{\top}(t-u)} .
$$

The matrix-valued stochastic integral in the second term of $Z_{j}(t)$ is understood as follows. For two $M_{d}(\mathbb{R})$-valued bounded and measurable functions $E(u)$ and $F(u)$ on $[t, s]$, the notation 
$\int_{s}^{t} E(u) \mathrm{d} \widetilde{L}(u) F(u)$ means that the matrix $G(s, t) \in M_{d}(\mathbb{R})$ with coordinates defined by

$$
G_{i j}(s, t)=\sum_{k=1}^{d} \sum_{l=1}^{d} \int_{s}^{t} E_{i k}(u) F_{l j}(u) \mathrm{d} \widetilde{L}_{k l}(u) .
$$

Here, $\widetilde{L}$ is the generic notation for some $\widetilde{L}_{j}$. We note that, since the $\widetilde{L}_{j}$ are supposed to be right continuous with left limits (RCLL), the processes $Z_{j}$ are also RCLL.

In energy markets such as gas and electricity it is often observed that a spike and an increase in volatility occur at the same time. This is known as the inverse leverage effect. To model this phenomena, we take the vector-valued subordinators $L_{i}$ driving the processes $Y_{i}, i=1, \ldots, m$, as the diagonal entries of the first $m$ matrix-valued subordinators $\widetilde{L}_{j}, j=1, \ldots, m$. If one of the off-diagonal elements jumps, the diagonal element also has to jump in order to keep the volatility process $\Sigma(t)$ in the positive-definite cone $\mathbb{S}_{d}^{+}$. Such a modeling choice ensures that the volatility jumps simultaneously with a spike in the spot price process. Since $n \geq m \in \mathbb{N}$, and the volatility process is a weighted sum of $n$ different volatility processes, there are still $n-m$ volatility processes $Z_{j}, j=m+1, \ldots, n$, which can be freely chosen.

By turning off the processes $Y_{i}$ we obtain a multivariate extension of the Schwartz model with stochastic volatility and stock price dynamics:

$$
S(t)=\Lambda(t) \cdot \exp (X(t))
$$

where $X(t)$ is defined in (2). The Schwartz model with constant volatility is a mean-reversion process proposed by Schwartz [18] for spot price dynamics in commodity markets like oil.

We impose the following log-integrability conditions on the subordinators: for $j=1, \ldots, n$, it holds that

$$
\mathbb{E}\left[\log ^{+}\left\|\tilde{L}_{j}(1)\right\|\right]<\infty,
$$

where $\log ^{+}(x)$ is defined as $\max (\log (x), 0)$. We use the Frobenius norm for matrices, $\|A\|=$ $\operatorname{tr}\left(A^{\top} A\right)^{1 / 2}, A \in M_{d}(\mathbb{R})$. This is the basic condition on the spot price model in [6].

For a detailed analysis of this spot price model for cross-commodity energy markets, we refer the reader to [6].

\section{Forward pricing}

In commodity markets, forward contracts are commonly traded on exchanges, including power, gas, oil, coal, etc. In this section we derive the forward price dynamics based on the multivariate spot price model (1).

Appealing to general arbitrage theory, we define the forward price $F(t, \tau)$ at time $t \geq 0$ for contracts delivering the energy commodity at time $\tau \geq t$ by (see, e.g. [9, Chapter 8B])

$$
F(t, \tau)=\mathbb{E}_{\mathbb{Q}}\left[S(\tau) \mid \mathcal{F}_{t}\right]
$$

where $\mathbb{Q}$ is a risk-neutral probability measure. This definition is valid as long as $S(\tau) \in L^{1}(\mathbb{Q})$. Below we give sufficient conditions ensuring integrability of the spot price with respect to a parametric class of pricing measures $\mathbb{Q}$. Since the spot price is an adapted process, we obtain the well-known convergence of spot and forward prices at maturity, i.e.

$$
F(\tau, \tau)=S(\tau) .
$$

It is worth noting that in some energy markets the forward contracts deliver the underlying commodity over a period rather than at a fixed maturity time $\tau$. This includes gas and electricity, 
but also more exotic markets like temperature. In these markets, the forward prices can be represented as some functional of $F(t, \tau)$, usually the average of $F(t, \tau)$ over $\tau$, taken over the delivery period of the forward contract. We will not consider this situation here; however, the calculations can be easily adjusted to take this into account (see, for example, [7] for a discussion).

The stochastic volatility model we are discussing gives rise to an incomplete market, and, hence, there exists a continuum of equivalent martingale measures $\mathbb{Q}$ that can be used for pricing. Moreover, in energy markets, the underlying spot is in general not tradeable, due to, for example, high storage costs, illiquidity, and other frictions such as transportation for delivery. In the extreme case of electricity, it is impossible to trade the underlying spot by the very nature of the commodity. Hence, the classical buy-and-hold hedging argument to pin down a forward price fails. As a result, all equivalent measures $\mathbb{Q} \sim P$ may be chosen as pricing measures since the underlying spot is not directly tradeable. In our considerations, we do not require the martingale property under $\mathbb{Q}$ for discounted spot prices. We refer the reader to [7] for more on this.

\subsection{A class of equivalent probabilities}

A convenient way to define a parametric class of risk-neutral probabilities for Lévy-based models is the Esscher transform (see [7] for applications of the Esscher transform in energy markets). Before introducing the measure transform, we need to introduce some notation and state some conditions: for $V \in \mathbb{S}_{d}^{+}$, we let $\phi_{\widetilde{L}_{j}}(V)$ be the cumulant function of $\widetilde{L}_{j}(1)$, that is,

$$
\phi_{\widetilde{L}_{j}}(V)=\ln \mathbb{E}\left[\exp \left(\operatorname{itr}\left(V \widetilde{L}_{j}(1)\right)\right)\right] .
$$

The Esscher transform is defined via the logarithmic moment generating functions of $\widetilde{L}_{j}$, and for this purpose we need to have certain exponential moments existing for $\widetilde{L}_{j}$. Let $\Theta_{j} \in \mathbb{S}_{d}^{+}$, and suppose that $\phi_{\tilde{L}_{j}}\left(-\mathrm{i} \Theta_{j}\right)$ is well defined. We have

$$
\phi_{\widetilde{L}_{j}}\left(-\mathrm{i} \Theta_{j}\right)=\int_{\mathbb{S}_{d}^{+}}\left\{\mathrm{e}^{\operatorname{tr}\left(\Theta_{j} U\right)}-1\right\} v_{\widetilde{L}_{j}}(\mathrm{~d} U),
$$

and, therefore, $\phi_{\widetilde{L}_{j}}\left(-\mathrm{i} \Theta_{j}\right)$ is well defined as long as

$$
\int_{\mathbb{S}_{d}^{+}}\left\{\mathrm{e}^{\operatorname{tr}\left(\Theta_{j} U\right)}-1\right\} v_{\widetilde{L}_{j}}(\mathrm{~d} U)<\infty .
$$

Note that, for $U, V \in \mathbb{S}_{d}^{+}, \operatorname{tr}(U V)=\langle U, V\rangle$, the inner product associated with the Frobenius matrix norm $\|A\|:=\operatorname{tr}\left(A^{\top} A\right)^{1 / 2}$. Hence, we have the inequality $|\operatorname{tr}(U V)| \leq\|U\|\|V\|$. Thus, a sufficient condition for (6) to hold is that

$$
\int_{\mathbb{S}_{d}^{+}} \mathrm{e}^{\left|\operatorname{tr}\left(\Theta_{j} U\right)\right|} v_{\widetilde{L}_{j}}(\mathrm{~d} U) \leq \int_{\mathbb{S}_{d}^{+}} \mathrm{e}^{\left\|\Theta_{j}\right\|\|U\|} v_{\widetilde{L}_{j}}(\mathrm{~d} U)<\infty .
$$

Throughout this paper, we suppose that there exists a constant $c_{j}>0$ such that the following exponential integrability condition holds for $v_{\widetilde{L}_{j}}$ :

$$
\int_{\mathbb{S}_{d}^{+}} \mathrm{e}^{c_{j}\|U\|} v_{\tilde{L}_{j}}(\mathrm{~d} U)<\infty
$$

for $j=1, \ldots, n$. This condition implies that $\phi_{\widetilde{L}_{j}}\left(-\mathrm{i} \Theta_{j}\right)$ is well defined for all $\Theta_{j} \in \mathbb{S}_{d}^{+}$such that $\left\|\Theta_{j}\right\| \leq c_{j}$. 
We move on to define the equivalent probability measure $\mathbb{Q}$. For $\Theta_{j} \in \mathbb{S}_{d}^{+}$, such that $\left\|\Theta_{j}\right\| \leq c_{j}$, define the processes

$$
\mathcal{V}_{j}(t)=\exp \left(\operatorname{tr}\left(\Theta_{j} \widetilde{L}_{j}(t)\right)-\phi_{\widetilde{L}}^{j}\left(-\mathrm{i} \Theta_{j}\right) t\right)
$$

for $j=1, \ldots, n$ and $t \leq \widetilde{T}$. Here we recall that $\widetilde{T}$ is a finite time horizon of the market for which all delivery times $\tau$ of interest are included. Note that $V_{j}(t)$ are martingales for $j=1, \ldots, m$ : in fact, by the exponential moment condition in (7) we find that

$$
\mathbb{E}\left[\mathcal{V}_{j}(t)\right]=1
$$

for every $j=1, \ldots, n$. For a vector $\theta_{0} \in \mathbb{R}^{d}$, introduce the process

$$
\mathcal{V}_{0}(t)=\exp \left(-\int_{0}^{t} \theta_{0}^{\top} \Sigma^{-1 / 2}(s) \mathrm{d} W(s)-\frac{1}{2} \theta_{0}^{\top} \int_{0}^{t} \Sigma^{-1}(s) \theta_{0} \mathrm{~d} s\right) .
$$

We have the following lemma.

Lemma 1. For all $\theta_{0} \in \mathbb{R}^{d}$, the process $\mathcal{V}_{0}(t)$ for $t \leq \tilde{T}$ is a martingale.

Proof. We show that the Novikov condition holds. From (5) we have, for every $j=1, \ldots, n$ and any $x \in \mathbb{R}^{d}$,

$$
\begin{aligned}
x^{\top} Z_{j}(t) x & =x^{\top} \mathrm{e}^{C_{j} t} Z_{j}(0) \mathrm{e}^{C_{j}^{\top} t} x+x^{\top} \int_{0}^{t} \mathrm{e}^{C_{j}(t-u)} \mathrm{d} \widetilde{L}_{j}(u) \mathrm{e}^{C_{j}^{\top}(t-u)} x \\
& \geq x^{\top} \mathrm{e}^{C_{j}(t-s)} Z_{j}(s) \mathrm{e}^{C_{j}^{\top}(t-s)} x
\end{aligned}
$$

by positive definiteness of the stochastic integral term. Hence,

$$
\Sigma(t)=\sum_{j=1}^{n} \omega_{j} Z_{j}(t) \geq \sum_{j=1}^{n} \omega_{j} \mathrm{e}^{C_{j}(t-s)} Z_{j}(s) \mathrm{e}^{C_{j}^{\top}(t-s)}>0 .
$$

But then, from linear algebra on positive-definite matrices,

$$
\Sigma^{-1}(t) \leq\left(\sum_{j=1}^{n} \omega_{j} \mathrm{e}^{-C_{j} t} Z_{j}^{-1}(0) \mathrm{e}^{-C_{j}^{\top} t}\right)^{-1},
$$

which means in particular that

$$
\theta_{0}^{\top} \Sigma^{-1}(t) \theta_{0} \leq \theta_{0}^{\top}\left(\sum_{j=1}^{n} \omega_{j} \mathrm{e}^{-C_{j} t} Z_{j}^{-1}(0) \mathrm{e}^{-C_{j}^{\top} t}\right)^{-1} \theta_{0} .
$$

As the right-hand side is a continuous function in $t$ on $[0, \widetilde{T}]$, it follows that

$$
\begin{aligned}
& \mathbb{E}\left[\exp \left(\frac{1}{2} \int_{0}^{\widetilde{T}} \theta_{0}^{\top} \Sigma^{-1}(t) \theta_{0} \mathrm{~d} t\right)\right] \\
& \leq \mathbb{E}\left[\exp \left(\frac{1}{2} \int_{0}^{\widetilde{T}} \theta_{0}^{\top}\left(\sum_{j=1}^{n} \omega_{j} \mathrm{e}^{-C_{j} t} Z_{j}^{-1}(0) \mathrm{e}^{-C_{j}^{\top} t}\right)^{-1} \theta_{0} \mathrm{~d} t\right)\right] \\
& \quad<\infty .
\end{aligned}
$$

Hence, by Novikov's condition, it follows from the Girsanov theorem that $\mathcal{V}_{0}(t)$ is a martingale. This completes the proof. 
Thus, the process

$$
\mathcal{V}(t)=\mathcal{V}_{0}(t) \times \mathcal{V}_{1}(t) \times \cdots \times \mathcal{V}_{n}(t)
$$

becomes a martingale for $t \leq \widetilde{T}$ and is the density process of a probability measure $\mathbb{Q}$ equivalent with $\mathbb{P}$, that is,

$$
\left.\frac{\mathrm{d} \mathbb{Q}}{\mathrm{d} \mathbb{P}}\right|_{\mathcal{F}_{t}}=\mathcal{V}(t) .
$$

From Girsanov's theorem we find that

$$
\mathrm{d} \widehat{W}(t)=\mathrm{d} W(t)-\Sigma^{-1 / 2}(t) \theta_{0} \mathrm{~d} t
$$

is an $\mathbb{R}^{d}$-valued Brownian motion with respect to $\mathbb{Q}$ on $t \in[0, \widetilde{T}]$. Furthermore, $\widetilde{L}_{j}(t)$ is a matrix-valued subordinator with respect to $\mathbb{Q}$, with characteristics stated in the following lemma.

Lemma 2. Assume that $\Theta_{j} \in \mathbb{S}_{d}^{+}$such that $\left\|\Theta_{j}\right\| \leq c_{j}$ for $j=1, \ldots, n$. Then the $\widetilde{L}_{j}(t)$ are subordinators under $\mathbb{Q}$ defined in (8) having Lévy measure with respect to $\mathbb{Q}$ given by

$$
\nu_{\widetilde{L}_{j}}^{\mathbb{Q}}(\mathrm{d} U)=\exp \left(\operatorname{tr}\left(\Theta_{j} U\right)\right) v_{\widetilde{L}_{j}}(\mathrm{~d} U)
$$

for $j=1, \ldots, n$.

Proof. First we prove that $\widetilde{L}_{j}(t)$ is a matrix-valued subordinator under $\mathbb{Q}$. Consider its conditional cumulant function with respect to $\mathbb{Q}, \widetilde{\phi}_{\widetilde{L}_{j}}^{(s, t)}(V)$ : for $0 \leq s \leq t$ and using Bayes' formula for conditional expectations (see [15]),

$$
\begin{aligned}
\widetilde{\phi}_{\widetilde{L}_{j}}^{(s, t)}(V) & =\ln \mathbb{E}_{\mathbb{Q}}\left[\exp \left(\operatorname{itr}\left(V\left(\widetilde{L}_{j}(t)-\widetilde{L}_{j}(s)\right)\right)\right) \mid \mathcal{F}_{s}\right] \\
& =\ln \mathbb{E}\left[\exp \left(\operatorname{itr}\left(V\left(\widetilde{L}_{j}(t)-\widetilde{L}_{j}(s)\right)\right)\right) \frac{\mathcal{V}(t)}{\mathcal{V}(s)} \mid \mathcal{F}_{s}\right] \\
& =\ln \mathbb{E}\left[\exp \left(\operatorname{itr}\left(\left(V-\mathrm{i} \Theta_{j}\right) \widetilde{L}_{j}(1)\right)\right) \mid \mathcal{F}_{s}\right]-\phi_{\widetilde{L}_{j}}\left(-\mathrm{i} \Theta_{j}\right)(t-s) \\
& =\ln \mathbb{E}\left[\exp \left(\mathrm{itr}\left(\left(V-\mathrm{i} \Theta_{j}\right) \widetilde{L}_{j}(1)\right)\right)\right]-\phi_{\widetilde{L}_{j}}\left(-\mathrm{i} \Theta_{j}\right)(t-s) \\
& =\phi_{\widetilde{L}_{j}}\left(V-\mathrm{i} \Theta_{j}\right)(t-s)-\phi_{\widetilde{L}_{j}}\left(-\mathrm{i} \Theta_{j}\right)(t-s) .
\end{aligned}
$$

In the second to last equality $\underset{\widetilde{\sim}}{\sim}$ used the independent increment property of $\widetilde{L}_{j}(t)$. This proves that the increment $\widetilde{L}_{j}(t)-\widetilde{L}_{j}(s)$ is stationary and independent of $\widetilde{\mathcal{F}}_{s}$, and, hence, a Lévy process with respect to the probability $\mathbb{Q}$. Moreover, $\widetilde{L}_{j}(t)$ has values in $\mathbb{S}_{d}^{+}$, and, therefore, it is a subordinator under $\mathbb{Q}$. From the above calculation we find its cumulant under $\mathbb{Q}$ to be

$$
\begin{aligned}
\widetilde{\phi}_{\widetilde{L}_{j}} & :=\ln \mathbb{E}_{\mathbb{Q}}\left[\exp \left(\mathrm{itr}\left(V \widetilde{L}_{j}(1)\right)\right)\right] \\
& =\phi_{\widetilde{L}_{j}}\left(V-\mathrm{i} \Theta_{j}\right)-\phi_{\widetilde{L}_{j}}\left(-\mathrm{i} \Theta_{j}\right) \\
& =\int_{\mathbb{S}_{d}^{+}}\left\{\mathrm{e}^{\mathrm{i} \operatorname{tr}\left(\left(V-\mathrm{i} \Theta_{j}\right) U\right)}-1\right\} v_{\tilde{L}_{j}}(\mathrm{~d} U)-\int_{\mathbb{S}_{d}^{+}}\left\{\mathrm{e}^{\mathrm{i} \operatorname{tr}\left(\left(-\mathrm{i} \Theta_{j}\right) U\right)}-1\right\} v_{L_{j}}(\mathrm{~d} U) \\
& =\int_{\mathbb{S}_{d}^{+}}\left\{\mathrm{e}^{\mathrm{itr}(V U)}-1\right\} \mathrm{e}^{\operatorname{tr}\left(\Theta_{j} U\right)} v_{\widetilde{L}_{j}}(\mathrm{~d} U) .
\end{aligned}
$$

This completes the proof. 
Since a subordinator is a pure jump process, the $\widetilde{L}_{j}$ for $j=1, \ldots, m$ must be independent of $\widehat{W}$ with respect to $\mathbb{Q}$, as a Brownian motion has continuous paths.

The parameters $\theta_{0}$ and $\Theta_{j}, j=1, \ldots, n$, may be referred to as the market prices of risk, extending the similar notion in the univariate case (see [7]). Note that the Esscher transform gives an exponential tilting of the Lévy measure of the matrix-valued subordinators $\widetilde{L}_{j}$. One effect of this is that the probabilities for large jumps are rescaled, and we may get more or less pronounced large jumps under $\mathbb{Q}$.

The dynamics of $X(t)$ under $\mathbb{Q}$ are given by

$$
\begin{aligned}
\mathrm{d} X(t) & =A X(t)+\Sigma^{1 / 2}(t)\left(\mathrm{d} \widehat{W}(t)+\Sigma^{-1 / 2}(t) \theta_{0} \mathrm{~d} t\right) \\
& =\left(\theta_{0}+A X(t)\right) \mathrm{d} t+\Sigma^{1 / 2}(t) \mathrm{d} \widehat{W}(t) .
\end{aligned}
$$

Thus, under $\mathbb{Q}$, the mean-reversion level is shifted from 0 to $\theta_{0}$. If $e_{k}^{\top} \theta_{0}>0$ for a $k=1, \ldots, d$ and $e_{k}$ being the $k$ th canonical unit vector of $\mathbb{R}^{d}$, then the base component of the $k$ th commodity mean reverts towards a higher level under $\mathbb{Q}$ than under $\mathbb{P}$, implying that the market assesses the base component as being more risky under the pricing measure $\mathbb{Q}$. A negative market price of risk $e_{k}^{\top} \theta_{0}$ will imply less risk loading on the $k$ th base component. The dynamics of $Y_{i}$ and $Z_{j}$ are changed in a similar fashion. We have, for $i=1, \ldots, m$,

$$
\begin{aligned}
\mathrm{d} Y_{i}(t) & =\left(\mu_{i}+B_{i} Y_{i}(t)\right) \mathrm{d} t+\eta_{i} \mathrm{~d} L_{i}(t) \\
& =\left(\mu_{i}+\eta_{i} \mathbb{E}_{\mathbb{Q}}[L(1)]+B_{i} Y_{i}(t)\right) \mathrm{d} t+\eta_{i} \mathrm{~d} L_{i}^{Q}(t),
\end{aligned}
$$

where $\mathrm{d} L_{i}^{\mathbb{Q}}(t):=\mathrm{d} L_{i}(t)-\mathbb{E}_{\mathbb{Q}}\left[L_{i}(1)\right] \mathrm{d} t$ is a $\mathbb{Q}$-martingale. Hence, the process $Y_{i}$ varies around the level $\mu_{i}+\eta_{i} \mathbb{E}_{\mathbb{Q}}\left[L_{i}(1)\right]$ under $\mathbb{Q}$, whereas the level is $\mu_{i}+\eta_{i} \mathbb{E}\left[L_{i}(1)\right]$ under $\mathbb{P}$. Thus, by appropriately choosing $\Theta_{i}$ we can obtain a higher or lower mean-reversion level, implying a higher or lower risk loading on the spike processes $Y_{i}$ under $\mathbb{Q}$. Similar considerations hold for the volatility processes $Z_{j}$. We remark in passing that the market prices of risk $\theta_{0}, \Theta_{1}, \ldots, \Theta_{n}$ will implicitly model the risk premium in the market, being the difference between the forward price and the predicted spot at delivery.

\subsection{Analysis of forward prices}

Before we derive the forward price, we need to introduce some notation and prove an auxiliary result. To this end, let $J_{d}$ be the linear operator that maps a vector $v \in \mathbb{R}^{d}$ to a symmetric $d \times d$ matrix $J_{d}(v)$, consisting of 0 s except on the diagonal, which is equal to $v$. On the other hand, diag is a linear operator mapping a matrix into a vector, where the vector is the diagonal of the matrix. The family of linear operators $\mathcal{C}_{j}(t)$ for $t \in[0, \widetilde{T}]$ are defined as

$$
\mathcal{C}_{j}(t): X \mapsto \omega_{j}\left[\left(\boldsymbol{C}_{j}-\boldsymbol{A}\right)^{-1}\left(\mathrm{e}^{C_{j} t} X \mathrm{e}^{C_{j}^{\top} t}-\mathrm{e}^{A t} X \mathrm{e}^{A^{\top} t}\right)\right],
$$

for $j=1, \ldots, n$. For $A$ being an $n \times n$ matrix, we denote the operator $\boldsymbol{A}$ associated with the matrix $A$ as $A: X \mapsto A X+X A^{\top}$. This operator can be represented as $\mathrm{vec}^{-1} \circ\left(\left(A \otimes I_{n}\right)+\right.$ $\left.\left(I_{n} \otimes A\right)\right) \circ v e c$, with $I_{n}$ being the $n \times n$ identity matrix and vec meaning the operator which stacks the columns of a matrix into a vector. Its inverse is denoted by $\boldsymbol{A}^{-1}$, which exists whenever $I_{n} \otimes A+A \otimes I_{n}$ is invertible. In this case, we can represent $A^{-1}$ by $\operatorname{vec}^{-1} \circ\left(\left(A \otimes I_{n}\right)+\right.$ $\left.\left(I_{n} \otimes A\right)\right)^{-1} \circ$ vec. Note that $A \otimes I_{n}+I_{n} \otimes A$ is equal to the Kronecker sum of the matrix $A$ with itself.

The following auxiliary result is useful in deriving the forward prices, and is proved in [6]. 
Lemma 3. Define $f(s, t):=\int_{s}^{t} \mathrm{e}^{A(t-u)} \Sigma(u) \mathrm{e}^{A^{\top}(t-u)} \mathrm{d} u$. Assume for $j=1, \ldots, n$ that $A$ and $C_{j}$ commute and the $\boldsymbol{A}-\boldsymbol{C}_{j}$ are invertible. Then it holds that

$$
f(s, t)=\sum_{j=1}^{n} \mathcal{C}_{j}(t-s) Z_{j}(s)+\int_{s}^{t} \mathcal{C}_{j}(t-v) \mathrm{d} \widetilde{L}_{j}(v)
$$

for $0 \leq s \leq t$.

Proof. The proof of this result is found in [6]. We include it here for the convenience of the reader. Using (5) and the assumption that $A$ and $C_{j}$ commute for $j=1, \ldots, n$, it holds that

$$
\begin{aligned}
f(s, t)= & \int_{s}^{t} \mathrm{e}^{A(t-u)} \sum_{j=1}^{n} \omega_{j}\left(\mathrm{e}^{C_{j}(u-s)} Z_{j}(s) \mathrm{e}^{C_{j}^{\top}(u-s)}+\int_{s}^{u} \mathrm{e}^{C_{j}(u-v)} \mathrm{d} \tilde{L}_{j}(v) \mathrm{e}^{C_{j}^{\top}(u-v)}\right) \\
& \times \mathrm{e}^{A^{\top}(t-u)} \mathrm{d} u \\
= & \sum_{j=1}^{n} \omega_{j} \int_{s}^{t} \mathrm{e}^{\left(C_{j}-A\right) u} \mathrm{e}^{A t-C_{j} s}\left(Z_{j}(s)+\int_{s}^{u} \mathrm{e}^{-C_{j} v} \mathrm{~d} \tilde{L}_{j}(v) \mathrm{e}^{-C_{j}^{\top} v}\right) \\
= & \sum_{j=1}^{n} \omega_{j}\left(\boldsymbol{C}_{j}-\boldsymbol{A}\right)^{-1}\left(\mathrm{e}^{C_{j}(t-s)} Z_{j}(s) \mathrm{e}^{C_{j}^{\top}(t-s)}-\mathrm{e}^{A(t-s)} Z_{j}(s) \mathrm{e}^{A^{\top}(t-s)}\right) \\
& +\int_{s}^{t} \int_{s}^{u}\left\{\mathrm{e}^{\left(C_{j}-A\right) u} \mathrm{e}^{A t} \mathrm{e}^{-C_{j} v} \mathrm{~d} \widetilde{L}_{j}(v) \mathrm{e}^{-C_{j}^{\top} v} \mathrm{e}^{A^{\top} t} \mathrm{e}^{\left(C_{j}-A\right)^{\top} u}\right\} \mathrm{d} u .
\end{aligned}
$$

The last integral is interpreted as first integrating with respect to $\mathrm{d} \widetilde{L}_{j}(v)$, and then integrating the obtained expression with respect to $\mathrm{d} u$. But, by spelling out the integrals in terms of sums, using the definition of the $\mathrm{d} \widetilde{L}_{j}(v)$ integrals, and invoking the stochastic Fubini theorem (see [17]), we obtain

$$
\begin{aligned}
& \int_{s}^{t} \int_{s}^{u}\left\{\mathrm{e}^{\left(C_{j}-A\right) u} \mathrm{e}^{A t} \mathrm{e}^{-C_{j} v} \mathrm{~d} \widetilde{L}_{j}(v) \mathrm{e}^{-C_{j}^{\top} v} \mathrm{e}^{A^{\top} t} \mathrm{e}^{\left(C_{j}-A\right)^{\top} u}\right\} \mathrm{d} u \\
& \quad=\int_{s}^{t} \int_{v}^{t}\left\{\mathrm{e}^{\left(C_{j}-A\right) u} \mathrm{e}^{A t} \mathrm{e}^{-C_{j} v} \mathrm{~d} \widetilde{L}_{j}(v) \mathrm{e}^{-C_{j}^{\top} v} \mathrm{e}^{A^{\top} t} \mathrm{e}^{\left(C_{j}-A\right)^{\top} u}\right\} \mathrm{d} u .
\end{aligned}
$$

Here, the right-hand side is interpreted as first integrating with respect to $\mathrm{d} u$, treating $\mathrm{d} \widetilde{L}_{j}(v)$ as a matrix and not a differential, and then integrating with respect to $\mathrm{d} \widetilde{L}_{j}(v)$ the obtained expression. Hence, we find that

$$
\begin{aligned}
f(s, t)= & \sum_{j=1}^{n} \mathcal{C}_{j}(t-s) Z_{j}(s) \\
& +\left(\boldsymbol{C}_{j}-\boldsymbol{A}\right)^{-1}\left(\int_{s}^{t} \mathrm{e}^{C_{j}(t-v)} \mathrm{d} \widetilde{L}_{j}(v) \mathrm{e}^{C_{j}^{\top}(t-v)}-\int_{s}^{t} \mathrm{e}^{A(t-v)} \mathrm{d} \widetilde{L}_{j}(v) \mathrm{e}^{A^{\top}(t-v)}\right) .
\end{aligned}
$$

This completes the proof.

By $\mathcal{C}_{j}^{*}(u)$ we mean the adjoint operator of $\mathcal{C}_{j}(u)$. Since $\mathcal{C}_{j}(u)$ is a linear operator on $d \times d$ matrices, we can represent it via a $d^{2} \times d^{2}$ matrix $\mathcal{K}_{j}(u)$ by $\mathcal{C}_{j}(u)=\operatorname{vec}^{-1} \circ \mathcal{K}_{j}(u) \circ$ vec. Hence, the adjoint $\mathcal{C}_{j}^{*}(u)$ has the representation $\mathcal{C}_{j}^{*}(u)=\operatorname{vec}^{-1} \circ \mathcal{K}_{j}^{T}(u) \circ$ vec.

We are now in the position to state the forward price. 
Proposition 1. For $k=1, \ldots, d$, suppose that the $\Theta_{j}$ are such that

$$
\sup _{u \in[0, \widetilde{T}]}\left\|\frac{1}{2} \mathcal{C}_{j}^{*}(u)\left(e_{k} e_{k}^{\top}\right)\right\|+\left\|\Theta_{j}\right\| \leq c_{j}
$$

for $j=1, \ldots, n$, and

$$
\sup _{u \in[0, \widetilde{T}]}\left\|\frac{1}{2} \mathcal{C}_{i}^{*}(u)\left(e_{k} e_{k}^{\top}\right)+J_{d}\left(e_{k}^{\top} \mathrm{e}^{B_{i} u} \eta_{i}\right)\right\|+\left\|\Theta_{i}\right\| \leq c_{i}
$$

for $i=1, \ldots, m$. Assume for $j=1, \ldots, n$ that $A$ and $C_{j}$ commute and the $\boldsymbol{A}-\boldsymbol{C}_{j}$ are invertible. Then the forward price at time $t \geq 0$ of a contract delivering the $d$ spots $S(\tau)$ at time $\tau \geq t$ is

$$
\begin{aligned}
F(t, \tau)=\Lambda(\tau) \cdot \exp ( & \mathrm{e}^{A(\tau-t)} X(t)+\sum_{i=1}^{m} \mathrm{e}^{B_{i}(\tau-t)} Y_{i}(t) \\
& +A^{-1}\left(I-\mathrm{e}^{A(\tau-t)}\right) \theta_{0}+\sum_{i=1}^{m} B_{i}^{-1}\left(I-\mathrm{e}^{B_{i}(\tau-t)}\right) \mu_{i} \\
& \left.+\frac{1}{2} \operatorname{diag}\left\{\sum_{j=1}^{n} \mathcal{C}_{j}(\tau-t) Z_{j}(t)\right\}\right) \cdot \Psi(\tau-t),
\end{aligned}
$$

where the kth coordinate of $\Psi(s) \in \mathbb{R}^{d}$ for $0 \leq s \leq \widetilde{T}$ is

$$
\begin{gathered}
\ln \Psi_{k}(s)=\sum_{j=1}^{n} \int_{0}^{s}\left\{\phi_{\widetilde{L}_{j}}\left(-\frac{1}{2} \mathrm{i} \mathcal{C}_{j}^{*}(u)\left(e_{k} e_{k}^{\top}\right)-\mathrm{i} \Theta_{j}\right)-\phi_{\widetilde{L}_{j}}\left(-\mathrm{i} \Theta_{j}\right)\right\} \mathrm{d} u \\
+\sum_{i=1}^{m} \int_{0}^{s}\left\{\phi_{\widetilde{L}_{i}}\left(-\frac{1}{2} \mathrm{i} \mathcal{C}_{i}^{*}(u)\left(e_{k} e_{k}^{\top}\right)-\mathrm{i} J_{d}\left(e_{k}^{\top} \mathrm{e}^{B_{i} u} \eta_{i}\right)-\mathrm{i} \Theta_{i}\right)\right. \\
\left.-\phi_{\widetilde{L}_{i}}\left(-\frac{1}{2} \mathrm{i}_{i}^{*}(u)\left(e_{k} e_{k}^{\top}\right)-\mathrm{i} \Theta_{i}\right)\right\} \mathrm{d} u
\end{gathered}
$$

for $k=1, \ldots, d$.

Proof. For simplicity, we let $m=n=1$ and omit the subscripts $i$ and $j$. From (3) and (4) along with the definition of the measure $\mathbb{Q}$, we have

$$
\begin{aligned}
X(\tau) & =\mathrm{e}^{A(\tau-t)} X(t)+\int_{t}^{\tau} \mathrm{e}^{A(\tau-u)} \Sigma^{1 / 2}(u) \mathrm{d} W(u) \\
& =\mathrm{e}^{A(\tau-t)} X(t)+\int_{t}^{\tau} \mathrm{e}^{A(\tau-u)} \theta_{0} \mathrm{~d} u+\int_{t}^{\tau} \mathrm{e}^{A(\tau-u)} \Sigma^{1 / 2}(u) \mathrm{d} \widehat{W}(u) \\
& =\mathrm{e}^{A(\tau-t)} X(t)+A^{-1}\left(I-\mathrm{e}^{A(\tau-t)}\right) \theta_{0}+\int_{t}^{\tau} \mathrm{e}^{A(\tau-u)} \Sigma^{1 / 2}(u) \mathrm{d} \widehat{W}(u)
\end{aligned}
$$

and

$$
Y(\tau)=\mathrm{e}^{B(\tau-t)} Y(t)+B^{-1}\left(I-\mathrm{e}^{B(\tau-t)}\right) \mu+\int_{t}^{\tau} \mathrm{e}^{B(\tau-u)} \eta \mathrm{d} L(u) .
$$


Hence, using the $\mathcal{F}_{t}$-adaptedness of $X(t)$ and $Y(t)$, we find that

$$
\begin{aligned}
F(t, \tau)= & \Lambda(\tau) \cdot \mathbb{E}_{\mathbb{Q}}\left[\exp (X(\tau)+Y(\tau)) \mid \mathcal{F}_{t}\right] \\
= & \Lambda(\tau) \cdot \exp \left(\mathrm{e}^{A(\tau-t)} X(t)+\mathrm{e}^{B(\tau-t)} Y(t)+A^{-1}\left(I-\mathrm{e}^{A(\tau-t)}\right) \theta_{0}\right. \\
& \left.\quad+B^{-1}\left(I-\mathrm{e}^{B(\tau-t)}\right) \mu\right) \\
& \cdot \mathbb{E}_{\mathbb{Q}}\left[\exp \left(\int_{t}^{\tau} \mathrm{e}^{A(\tau-u)} \Sigma^{1 / 2}(u) \mathrm{d} \widehat{W}(u)+\int_{t}^{\tau} \mathrm{e}^{B(\tau-u)} \eta \mathrm{d} L(u)\right) \mid \mathcal{F}_{t}\right] .
\end{aligned}
$$

We consider the expectation in the last equality, which we denote by $\widehat{F}(t, \tau)$. Let $g_{t, \tau}$ be the $\sigma$-algebra generated by $\mathcal{F}_{t}$ and $\widetilde{L}(u)$ for $t \leq u \leq \tau$. Recalling that, under $\mathbb{Q}, \widehat{W}$ and $\widetilde{L}$ are independent, we find from the tower property of the conditional expectation operator that

$$
\begin{aligned}
& \widehat{F}(t, \tau)=\mathbb{E}_{\mathbb{Q}} {\left[\mathbb{E}_{\mathbb{Q}}\left[\exp \left(\int_{t}^{\tau} \mathrm{e}^{A(\tau-s)} \Sigma^{1 / 2}(s) \mathrm{d} \widehat{W}(s)+\int_{t}^{\tau} \mathrm{e}^{B(\tau-s)} \eta \mathrm{d} L(s)\right) \mid g_{t, \tau}\right] \mid \mathcal{F}_{t}\right] } \\
&=\mathbb{E}_{\mathbb{Q}} {\left[\exp \left(\int_{t}^{\tau} \mathrm{e}^{B(\tau-s)} \eta \mathrm{d} L(s)\right)\right.} \\
&\left.\cdot \mathbb{E}_{\mathbb{Q}}\left[\exp \left(\int_{t}^{\tau} \mathrm{e}^{A(\tau-s)} \Sigma^{1 / 2}(s) \mathrm{d} \widehat{W}(s)\right) \mid g_{t, \tau}\right] \mid \mathcal{F}_{t}\right] \\
&=\mathbb{E}_{\mathbb{Q}}\left[\exp \left(\frac{1}{2} \operatorname{diag}\left[\int_{t}^{\tau} \mathrm{e}^{A(\tau-s)} \Sigma(s) \mathrm{e}^{A^{\top}(\tau-s)} \mathrm{d} s\right]+\int_{t}^{\tau} \mathrm{e}^{B(\tau-s)} \eta \mathrm{d} L(s)\right) \mid \widetilde{F}_{t}\right] .
\end{aligned}
$$

In the second equality we used the fact that $L$ is measurable with respect to $g_{t, \tau}$, while in the last equality we applied the facts that the Wiener integral of a deterministic function is independent of $\mathcal{F}_{t}$ and a Gaussian random variable.

From Lemma 3, we find after appealing to the $\mathcal{F}_{t}$-measurability of $Z(t)$ and the independent increment property of Lévy processes that

$$
\begin{aligned}
& \widehat{F}(t, \tau)= \mathbb{E}_{\mathbb{Q}}\left[\operatorname { e x p } \left(\frac{1}{2} \operatorname{diag}(\mathcal{C}(\tau-t) Z(t))+\frac{1}{2} \operatorname{diag}\left(\int_{t}^{\tau} \mathcal{C}(\tau-u) \mathrm{d} \widetilde{L}(u)\right)\right.\right. \\
&\left.\left.\quad+\int_{t}^{\tau} \mathrm{e}^{B(\tau-u)} \eta \mathrm{d} L(u)\right) \mid \mathcal{F}_{t}\right] \\
&=\exp \left(\frac{1}{2} \operatorname{diag}(\mathcal{C}(\tau-t) Z(t))\right) \\
& \cdot \mathbb{E}_{\mathbb{Q}}\left[\exp \left(\frac{1}{2} \operatorname{diag}\left(\int_{t}^{\tau} \mathcal{C}(\tau-u) \mathrm{d} \widetilde{L}(u)\right)+\int_{t}^{\tau} \mathrm{e}^{B(\tau-u)} \eta \mathrm{d} L(u)\right)\right] .
\end{aligned}
$$

Let us focus on the expectation above, and denote it by $\Psi(t, \tau)$. It is a vector in $\mathbb{R}^{d}$, and we look at it componentwise. Note that the $k$ th coordinate of $\operatorname{diag}\left(\int_{t}^{\tau} \mathcal{C}(\tau-u) \mathrm{d} \widetilde{L}(u)\right)$ can be expressed as $e_{k}^{\top} \int_{t}^{\tau} \mathcal{C}(\tau-u) \mathrm{d} \widetilde{L}(u) e_{k}$, while the $k$ th coordinate of $\int_{t}^{\tau} \mathrm{e}^{B(\tau-u)} \eta \mathrm{d} L(u)$ is $e_{k}^{\top} \int_{t}^{\tau} \mathrm{e}^{B(\tau-u)} \eta \mathrm{d} L(u)$. Hence, from the fundamental relation $w^{k} U w=\operatorname{tr}\left(w w^{k} A\right)$ for a vector $w$ and a matrix $U$,

$$
\begin{aligned}
\Psi_{k}(t, \tau) & =\mathbb{E}_{\mathbb{Q}}\left[\exp \left(\frac{1}{2} e_{k}^{\top} \int_{t}^{\tau} \mathcal{C}(\tau-u) \mathrm{d} \widetilde{L}(u) e_{k}+e_{k}^{\top} \int_{t}^{\tau} \mathrm{e}^{B(\tau-u)} \eta \mathrm{d} L(u)\right)\right] \\
& =\mathbb{E}_{\mathbb{Q}}\left[\exp \left(\operatorname{itr}\left(-\frac{1}{2} \mathrm{i} e_{k} e_{k}^{\top} \int_{t}^{\tau} \mathcal{C}(\tau-u) \mathrm{d} \widetilde{L}(u)\right)+\int_{t}^{\tau} e_{k} \mathrm{e}^{B(\tau-u)} \eta \mathrm{d} L(u)\right)\right] .
\end{aligned}
$$


Note that $e_{k}^{\top} \mathrm{e}^{B(\tau-u)} \eta$ is a $d$-dimensional vector. It is simple to see that

$$
\int_{t}^{\tau} e_{k}^{\top} \mathrm{e}^{B(\tau-u)} \eta \mathrm{d} L(u)=\operatorname{tr}\left(\int_{t}^{\tau} J_{d}\left(e_{k}^{\top} \mathrm{e}^{B(\tau-u)} \eta\right) \mathrm{d} \widetilde{L}(u)\right) .
$$

Hence,

$$
\begin{aligned}
\Psi_{k}(t, \tau)= & \mathbb{E}_{\mathbb{Q}}\left[\operatorname { e x p } \left(\operatorname{itr}\left(-\frac{1}{2} \mathrm{i} \int_{t}^{\tau} e_{k} e_{k}^{\top} \mathcal{C}(\tau-u) \mathrm{d} \widetilde{L}(u)\right)\right.\right. \\
& \left.\left.+\mathrm{itr}\left(-\mathrm{i} \int_{t}^{\tau} J_{d}\left(e_{k}^{\top} \mathrm{e}^{B(\tau-u)} \eta\right) \mathrm{d} \widetilde{L}(u)\right)\right)\right] \\
= & \mathbb{E}\left[\exp \left(\operatorname{itr}\left(\int_{t}^{\tau}\left\{-\frac{1}{2} \mathrm{i} e_{k} e_{k}^{\top} \mathcal{C}(\tau-u)-\mathrm{i} J_{d}\left(e_{k}^{\top} \mathrm{e}^{B(\tau-u)} \eta\right)\right\} \mathrm{d} \widetilde{L}(u)\right)\right)\right] \\
& \times \exp \left(-\phi_{\widetilde{L}}(-\mathrm{i} \Theta)\right) .
\end{aligned}
$$

Next, observe that the stochastic integral can be expressed as

$$
\begin{aligned}
\int_{t}^{\tau}\left\{\frac{1}{2} e_{k} e_{k}^{\top} \mathcal{C}(\tau-u)+J_{d}\left(e_{k}^{\top} \mathrm{e}^{B(\tau-u)} \eta\right)\right\} \mathrm{d} \widetilde{L}(u) \\
\quad=\lim _{\left|\Delta_{i}\right| \rightarrow 0} \sum_{i=0}^{n-1}\left\{\frac{1}{2} e_{k} e_{k}^{\top} \mathcal{C}\left(\tau-u_{i}\right)+J_{d}\left(e_{k}^{\top} \mathrm{e}^{B(\tau-u)}\right) \eta\right\} \Delta \widetilde{L}\left(u_{i}\right)
\end{aligned}
$$

for partitions $t=u_{0}<\cdots<u_{n}=\tau$ with $\Delta_{i}:=\widetilde{L}\left(u_{i+1}\right)-\widetilde{L}\left(u_{i}\right)$ and $\Delta_{i}:=u_{i+1}-u_{i}$. By the independence of increments of a Lévy process, and continuity of the exponential function together with Fubini-Tonelli's theorem, we obtain

$$
\begin{aligned}
& \mathbb{E}\left[\exp \left(\operatorname{itr}\left(\int_{t}^{\tau}\left\{-\frac{1}{2} \mathrm{i} e_{k} e_{k}^{\top} \mathcal{C}(\tau-u)-\mathrm{i} J_{d}\left(e_{k}^{\top} \mathrm{e}^{B(\tau-u)} \eta\right)\right\} \mathrm{d} \widetilde{L}(u)\right)\right)\right] \\
& \quad=\lim _{\left|\Delta_{i}\right| \rightarrow 0} \prod_{i=1}^{n-1} \mathbb{E}\left[\exp \left(\operatorname{itr}\left(\left\{-\frac{1}{2} \mathrm{i} e_{k} e_{k}^{\top} \mathcal{C}_{j}\left(\tau-u_{i}\right)-\mathrm{i} J_{d}\left(e_{k}^{\top} \mathrm{e}^{B\left(\tau-u_{i}\right)} \eta\right)\right\} \Delta \widetilde{L}\left(u_{i}\right)\right)\right)\right] .
\end{aligned}
$$

Now, the linear operators $\mathcal{C}\left(\tau-u_{i}\right)$ can be represented as vec ${ }^{-1} \circ \mathcal{K}\left(\tau-u_{i}\right) \circ$ vec for a matrix $\mathcal{K} \in \mathbb{R}^{d^{2} \times d^{2}}$. Hence, since for quadratic matrices $\operatorname{tr}(V X)=\operatorname{vec}(V)^{\top} \operatorname{vec}(X)$, we find that

$$
\begin{aligned}
\operatorname{tr}\left(\left(e_{k} e_{k}^{\top}\right) \mathcal{C}\left(\tau-u_{i}\right) \Delta \widetilde{L}\left(u_{i}\right)\right) & =\operatorname{vec}\left(e_{k} e_{k}^{\top}\right)^{\top} \operatorname{vec}\left(\mathcal{C}\left(\tau-u_{i}\right) \Delta \widetilde{L}\left(u_{i}\right)\right) \\
& =\operatorname{vec}\left(e_{k} e_{k}^{\top}\right)^{\top} \operatorname{vec}\left(\operatorname{vec}^{-1} \mathcal{K}\left(\tau-u_{i}\right) \operatorname{vec}\left(\Delta \widetilde{L}\left(u_{i}\right)\right)\right) \\
& =\operatorname{vec}\left(e_{k} e_{k}^{\top}\right)^{\top} \mathcal{K}\left(\tau-u_{i}\right) \operatorname{vec}\left(\Delta \widetilde{L}\left(u_{i}\right)\right) \\
& =\left(\mathcal{K}^{\top}\left(\tau-u_{i}\right) \operatorname{vec}\left(e_{k} e_{k}^{\top}\right)\right)^{\top} \operatorname{vec}\left(\Delta \widetilde{L}\left(u_{i}\right)\right) \\
& =\operatorname{tr}\left(\operatorname{vec}^{-1}\left(\mathcal{K}^{\top}\left(\tau-u_{i}\right) \operatorname{vec}\left(e_{k} e_{k}^{\top}\right)\right) \Delta \widetilde{L}\left(u_{i}\right)\right) \\
& =\operatorname{tr}\left(\mathcal{C}^{*}\left(\tau-u_{i}\right)\left(e_{k} e_{k}^{\top}\right) \Delta \widetilde{L}\left(u_{i}\right)\right)
\end{aligned}
$$

Thus,

$$
\begin{gathered}
\mathbb{E}\left[\exp \left(\operatorname{itr}\left(\left\{-\frac{1}{2} \mathrm{i} e_{k} e_{k}^{\top} \mathcal{C}_{j}\left(\tau-u_{i}\right)-\mathrm{i} J_{d}\left(e_{k}^{\top} \mathrm{e}^{B\left(\tau-u_{i}\right)} \eta\right)\right\} \Delta \widetilde{L}\left(u_{i}\right)\right)\right)\right] \\
=\exp \left(\phi_{L}\left(-\frac{1}{2} \mathrm{i} \mathcal{C}^{*}\left(\tau-u_{i}\right)\left(e_{k} e_{k}^{\top}\right)-\mathrm{i} J_{d}\left(e_{k} \mathrm{e}^{B\left(\tau-u_{i}\right)} \eta\right)\right) \Delta u_{i}\right)
\end{gathered}
$$


Nesting up, we find that

$$
\ln \Psi_{k}(t, \tau)=\int_{t}^{\tau}\left\{\phi_{\widetilde{L}}\left(-\frac{1}{2} \mathrm{i} \mathcal{C}^{*}(\tau-u)\left(e_{k} e_{k}^{\top}\right)-\mathrm{i} J_{d}\left(e_{k}^{\top} \mathrm{e}^{B(\tau-u)} \eta\right)-\mathrm{i} \Theta\right)-\phi_{\widetilde{L}}(-\mathrm{i} \Theta)\right\} \mathrm{d} u .
$$

By changing variables we see that $\Psi_{k}$ depends on $\tau-t$. This completes the proof.

The forward price $F(t, \tau)$ gives us the joint dynamics of forward prices on each of the spot commodities. Hence, it is a $d$-variate process, giving the cross-commodity forward price dynamics. Recall that '.' denotes the pointwise product, and that we use the notation for the exponential function interchangeably, in the sense that $\exp (x)$ means elementwise exponentiation as long as $x$ is a vector, and the matrix exponential when $x$ is a matrix.

Note that, since $\mathcal{C}_{j}(0)=0$ and $\Psi_{k}(0)=1$ for $k=1, \ldots, d$, it is easily seen that the expression for $F(t, \tau)$ is equal to $S(t)$ when $\tau=t$. This shows that the forward price converges to the spot at maturity, which it should by definition of the forward price as the conditional expectation of the spot at maturity. More interestingly, the forward price dynamics are explicitly dependent on the stochastic volatility factors $Z_{j}(t)$. This has the interesting effect that even in the case of no spike components in the spot dynamics (i.e. when $m=0$ ), the forward price dynamics will have jumps. That is, a continuous spot price dynamic with stochastic volatility will imply a forward price dynamic which jumps according to the jumps in the stochastic volatility.

We state the dynamics of the forward price.

Proposition 2. Suppose that the conditions of Proposition 1 hold. Then the dynamics of $F_{k}(t, \tau)$ of commodity $k$ with respect to $\mathbb{Q}$ are

$$
\begin{aligned}
\frac{\mathrm{d} F_{k}(t, \tau)}{F_{k}(t-, \tau)}= & e_{k}^{\top} \mathrm{e}^{A(\tau-t)} \Sigma^{1 / 2}(t) \mathrm{d} \widehat{W}(t) \\
& +\sum_{i=1}^{m} \int_{\mathbb{S}_{d}^{+} \backslash\{0\}}\left\{\exp \left(\frac{1}{2} e_{k}^{\top} \operatorname{diag}\left(\mathcal{C}_{i}(\tau-t) V\right)+e_{k}^{\top} \mathrm{e}^{B_{i}(\tau-t)} \eta_{i} \operatorname{diag}(V)\right)-1\right\} \\
& \times \widetilde{N}_{i}^{\mathbb{Q}}(\mathrm{d} t, \mathrm{~d} V) \\
& +\sum_{j=m+1}^{n} \int_{\mathbb{S}_{d}^{+} \backslash\{0\}}\left\{\exp \left(\frac{1}{2} e_{k}^{\top} \operatorname{diag}\left(\mathcal{C}_{j}(\tau-t) V\right)\right)-1\right\} \widetilde{N}_{j}^{\mathbb{Q}}(\mathrm{d} t, \mathrm{~d} V) .
\end{aligned}
$$

Here, $\widetilde{N}_{j}^{\mathbb{Q}}(\underset{\sim}{\mathrm{d} t}, \mathrm{~d} V)=N_{j}(\mathrm{~d} t, \mathrm{~d} V)-\exp \left(\operatorname{tr}\left(V \Theta_{j}\right)\right) \nu_{\widetilde{L}_{j}}(\mathrm{~d} V) \mathrm{d} t$ and $N_{j}$ is the Poisson random measure of $\widetilde{L}_{j}$ for $j=1, \ldots, n$.

Proof. First, note that, by definition, the process $t \mapsto F_{k}(t, \tau)$ is a martingale for $t \leq \tau$. From Proposition 1, we have, in a compact form,

$$
F_{k}(t, \tau)=\Lambda_{k}(\tau) \exp \left(e_{k}^{\top} \mathrm{e}^{A(\tau-t)} X(t)+e_{k}^{\top} \mathrm{e}^{B(\tau-t)} Y(t)+\frac{1}{2} \operatorname{diag}(\mathcal{C}(\tau-t) Z(t))\right) G_{k}(\tau-t),
$$

where we have collected all nonrandom terms into $G$, being a vector in $\mathbb{R}^{d}$. Since $F_{k}(t, \tau)$ depends on $X(t), Y(t)$, and $Z(t)$, the dynamics of $F_{k}$ will necessarily be expressible in terms of the $\mathbb{Q}$-Wiener process $\widehat{W}$ and the compensated Poisson random measures of $\widetilde{L}_{j}$ under $\mathbb{Q}$. Hence, when using Itô's formula for jump processes (see, e.g. [19]), we only need to focus on terms involving $\mathrm{d} \widehat{W}$ and $\widetilde{N}_{j}^{Q}(\mathrm{~d} t, \mathrm{~d} V)$. To do this, we note that the dynamics of $Y(t)$ can be written as

$$
\mathrm{d} Y(t)=(\mu+B Y(t)) \mathrm{d} t+\eta \mathrm{d}(\operatorname{diag}(\widetilde{L}(u))) .
$$


Moreover, since $\mathcal{C}(\tau-t)$ and diag are linear operators on matrices, $F_{k}$ is a function of linear combinations of $Z_{u, v}(t)$ and $Y_{u}(t)$ for $u, v=1, \ldots, d$. Hence, the dynamics will consist of linear combinations of the elements of the $\widetilde{L}(t)$ matrix. Applying Itô's formula, taking into account all these considerations, yields the result.

We see that there is a Samuelson effect in the forward price dynamics. The volatility appearing in the $\mathrm{d} \widehat{W}$-term of the dynamics takes the form $e_{k}^{\top} \exp (A(\tau-t)) \Sigma^{1 / 2}(t)$. The contribution from $e_{k}^{\top} \exp (A(\tau-t))$ is an 'exponential scaling' of the stochastic spot volatility $\Sigma^{1 / 2}(t)$. Moreover, as the time to maturity goes to 0 , we obtain a convergence of the forward volatility to the spot volatility:

$$
\lim _{\tau \downarrow t} e_{k}^{\top} \mathrm{e}^{A(\tau-t)} \Sigma^{1 / 2}(t)=\Sigma^{1 / 2}(t) .
$$

This yields a generalization of the Samuelson effect known in the one-dimensional case to cross-commodity forward prices. We note that the one-dimensional Samuelson effect gives a forward volatility which is exponential dampening (in 'time to maturity') of the spot volatility. However, in the multidimensional case, the shape of $e_{k}^{\top} \exp (A(\tau-t)) \Sigma^{1 / 2}$ will be much richer than simply exponential decay in time to maturity towards spot volatility. In fact, one may get situations where the forward volatility is increasing rather than decreasing with the time to maturity. For example, choosing $A$ to be a matrix of CARMA type (see [7]), we may get this situation, which is in contrast to the classical Samuelson effect. Observe also that the jump terms in the dynamics of the forward price contribute to the Samuelson effect; however, this is much more complex to analyse.

In the next proposition we show that the forward price will behave like the seasonal function in the long end of the market. To prove this result, we dispense with the restriction that the forward price is only defined up to maturities $\widetilde{T}<\infty$, but carry out an asymptotic consideration of $F$ focusing only on the expression in Proposition 1.

Proposition 3. Let $F(t, \tau)$ be given as in Proposition 1, and suppose that $\lim _{t \rightarrow \infty} \ln \Psi(t)$ exists. Then

$$
\lim _{\tau \rightarrow \infty}(\ln F(t, \tau)-\ln \Lambda(\tau))=A^{-1} \theta_{0}+\sum_{i=1}^{m} B_{i}^{-1} \mu_{i}+\lim _{\tau \rightarrow \infty} \ln \Psi(\tau) .
$$

Here, we understand the operations of the function ln coordinatewise.

Proof. This result follows immediately from the assumption that the real parts of the eigenvalues of the matrices $A, B_{i}$, and $C_{j}$ are all negative, $i=1, \ldots, m$ and $j=1, \ldots, n$.

Note that the condition that $\Psi(\tau)$ has a limit is equivalent to the existence of a stationary dynamic of $\int_{0}^{t} \mathcal{C}_{j}(t-s) \mathrm{d} \widetilde{L}_{j}(s)$ and $\int_{0}^{t} \mathrm{e}^{B_{i}(t-s)} \eta_{i} \mathrm{~d} L_{i}(u)$ under $\mathbb{Q}$. If this is the case then we can interpret $\lim _{\tau \rightarrow \infty} \ln \Psi(\tau)$ as the long-term mean value of the market price of risk.

From Proposition 3, contracts with maturities in the long end of the market will have forward prices which are basically equal to the seasonality function, adjusted by the stationary mean values of $Y_{i}$ and $Z_{j}$ and the market prices of risk, that is,

$$
F(t, \tau) \sim \text { constant } \cdot \Lambda(\tau) .
$$

As a result of mean reversion of the spot prices, the forward prices are not reacting to changes in the spot in the long end but only following the seasonal mean adjusted by the market prices of risk. 


\subsection{Shapes of the forward curve}

Note that we can view the forward price dynamics as a regression on the spot price, leverage terms, and the volatility processes. Introduce the shorthand notation $\Theta(t, \tau) \in \mathbb{R}^{d}$ given by

$$
\ln \Theta(t, \tau):=\ln \Psi(\tau-t)+\ln \Lambda(\tau)+A^{-1}\left(I-\mathrm{e}^{A(\tau-t)}\right) \theta_{0}+\sum_{i=1}^{m} B_{i}^{-1}\left(I-\mathrm{e}^{B_{i}(\tau-t)}\right) \mu_{i} .
$$

Then, from Proposition 1,

$$
\begin{aligned}
\ln F(t, \tau)= & \ln \Theta(t, \tau)+\mathrm{e}^{A(\tau-t)} \ln S(t)+\sum_{i=1}^{m}\left(\mathrm{e}^{B_{i}(\tau-t)}-\mathrm{e}^{A(\tau-t)}\right) Y_{i}(t) \\
& +\frac{1}{2} \operatorname{diag}\left\{\sum_{j=1}^{n} \mathcal{C}_{j}(\tau-t) Z_{j}(t)\right\}
\end{aligned}
$$

Here $\Theta$ is a level adjustment function. The impact of the various factors on the forward price $F(t, \tau)$ goes through the matrix exponentials. In fact, the forward price of one commodity depends on the normal variation processes $X$, spike processes $Y_{i}$, and volatility processes $Z_{j}$ of all the commodities modeled. Hence, for example, if one of the commodities has a spike then the forward prices of all the other commodities will be influenced. There is also a direct influence from the volatility processes between the forwards, both directly and indirectly via the stochastic volatility $\Sigma(t)$ in the dynamics of $X$.

As noted in Andresen et al. [1], the mean-reverting structure represented by a matrix exponential has a richer structure than in the one-dimensional case, and we may include hump structures in the forward curve. We discuss the potential shapes of $\tau \mapsto F(t, \tau)$ in more detail. Since $A \in \mathrm{GL}_{d}(\mathbb{R})$, it is diagonalizable. So it holds that $\mathrm{e}^{A(\tau-t)}=U \mathrm{e}^{V(\tau-t)} U^{-1}$, where $U$ is a basis of eigenvectors and $V$ is a matrix with the eigenvalues of $A$ on the diagonal and $0 \mathrm{~s}$ elsewhere (see, e.g. [12]). Hence, an entry of the vector $\mathrm{e}^{A(\tau-t)} X(t)$ can be represented as

$$
\sum_{i=1}^{d} a_{1 i} \mathrm{e}^{\lambda_{i}(\tau-t)} X_{1}(t)+\sum_{i=1}^{d} a_{2 i} \mathrm{e}^{\lambda_{i}(\tau-t)} X_{2}(t)+\cdots+\sum_{i=1}^{d} a_{d i} \mathrm{e}^{\lambda_{i}(\tau-t)} X_{d}(t)
$$

for some constants $a_{i j} \in \mathbb{R}$ and eigenvalues $\lambda_{i}, i, j=1, \ldots, d$. Consider first the Schwartz model with constant volatility, i.e. no contribution of the processes $Y_{i}$ and $Z_{j}$ in the forward price. If $X$ is positive in all its components, $\lambda_{i}$ is real, and $a_{i j} \in \mathbb{R}^{+}$for all $i, j=1, \ldots, d$, then the forward is in backwardation since the eigenvalues have negative real parts. The opposite conclusion (i.e. forward prices in contango) holds when $X$ is negative in all its components. A more realistic situation with this model is the case where there are humps in the forward curve and where the forward is changing between backwardation and contango over time. This behavior has been observed for real market prices. For example, on page 216 of [11] the forward curve of WTI oil is plotted together with the spot price. The shape of the curve varies over time from contango to backwardation, including positive humps in the short end. When the constants $a_{j i}$ for fixed $j$ are not all of the same sign and the entries of $X$ all have a positive sign, then an entry of $\mathrm{e}^{A(\tau-t)} X(t)$ is given by a linear combination of increasing and decreasing exponentials which rise and decay at different speeds. Owing to this, the forward may alternate between backwardation and contango and humps may appear (see Figure 1(a)). Another possibility is the case of complex eigenvalues. This leads to an oscillating structure in 


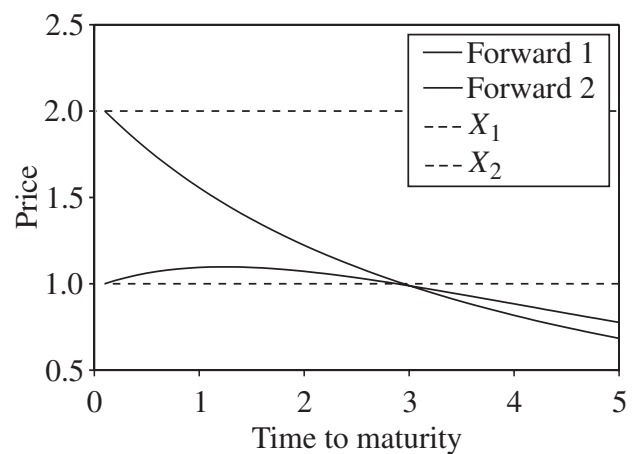

(a)

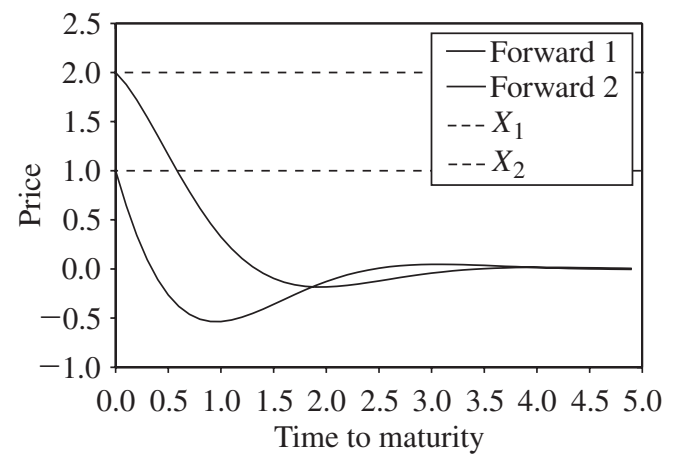

(b)

Figure 1: Paths of $\mathrm{e}^{A(\tau-t)} X$ for (a) real and (b) complex eigenvalues of $A$. Moreover, $X=(1,2)^{\top}$ is taken constant.

the forward curve. So a change upward in the $i$ th component of $X$ may cause a rise or fall of the forward depending on the time to maturity (see Figure 1(b)).

A similar analysis can be made for the spike processes $Y_{i}$. However, since $Y_{i}$ is a pure jump process, it will contribute to sudden changes in the forward curve. These humps may be upward or downward pointing depending on the time to maturity. The jumps caused by the spike process $Y_{i}$ may be averaged out by jumps in the volatility process $Z_{i}$. The processes $Y_{i}$ and $Z_{i}$ are driven by related subordinators $L_{i}$ and $\widetilde{L}_{i}$. Hence, $Y_{i}$ and $Z_{i}$ may have simultaneous jumps; however, depending on the value of the matrices $A, B_{i}$, and $C_{j}$, an upward jump caused by the volatility process $Z_{i}$ may simultaneously have a downward jump caused by the spike process $Y_{i}$. Hence, the jumps may average out. Conversely, depending on the parameters $A$, $B_{i}$, and $C_{j}$, the jumps in $Y_{i}$ and $Z_{i}$ may enlarge each other and lead to a big jump in the forward curve. This is a result of the inverse leverage effect in the spot model, which we see have a 'double' impact on forward prices.

\section{Transform-based pricing of options}

Spread options are popular derivatives in the energy market to hedge price differences. For instance, spread options are traded on the difference in electricity forward prices in neighboring markets, or on the difference between electricity and one of its fuels, including spark (electricity and gas) and dark (electricity and coal) spreads. On the New York Mercantile Exchange, options on spreads between forwards on different refined oils are traded.

In this section we will consider pricing of options on a combination of forwards, with the spread as a special case. The dynamics of the forward are given by our multivariate model, which allows for the application of the Fourier method to pricing.

Consider an option written on a combination of the forwards expressed via the payoff function $f: \mathbb{R}^{d} \mapsto \mathbb{R}$. At exercise time $T \leq \tau$, the option pays out $f(F(T, \tau))$, with the forwards maturing at time $\tau \geq T$. Supposing that $f(F(T, \tau))$ is integrable with respect to the pricing measure $\mathbb{Q}$ defined in the previous section, the option price at time $t \leq T$ becomes

$$
C(t)=\mathrm{e}^{-r(T-t)} \mathbb{E}_{\mathbb{Q}}\left[f(F(T, \tau)) \mid \mathcal{F}_{t}\right],
$$

where the constant $r>0$ is the risk-free interest rate. As it turns out, the forward price, or rather its logarithm, has a semianalytic cumulant function, which allows for the application 
of the Fourier method to option pricing (see [8] for a general treatment of Fourier methods in pricing of options). We now discuss this in more detail.

First, define the function

$$
g(x):=f\left(\mathrm{e}^{x}\right)
$$

and observe that

$$
g(\ln x)=f(x),
$$

where we have used pointwise exponentials and logarithms. Suppose that $g \in L^{1}\left(\mathbb{R}^{d}\right)$, the space of integrable functions on $\mathbb{R}^{d}$, and recall the $d$-dimensional Fourier transform

$$
\widehat{g}(y)=\int_{\mathbb{R}^{d}} g(x) \mathrm{e}^{-\mathrm{i}\langle x, y\rangle} \mathrm{d} x .
$$

If $\widehat{g} \in L^{1}\left(\mathbb{R}^{d}\right)$ then the inverse Fourier transform becomes

$$
g(x)=\frac{1}{2 \pi} \int_{\mathbb{R}^{d}} \widehat{g}(y) \mathrm{e}^{\mathrm{i}\langle y, x\rangle} \mathrm{d} y .
$$

See [10] for these definitions. To price options, let us introduce the conditional cumulant function of the log-forward prices under $\mathbb{Q}$ : for $s \leq t \leq \tau$ and $x \in \mathbb{R}^{d}$, define

$$
\widetilde{\phi}_{\ln F}^{(s, t, \tau)}(x):=\ln \mathbb{E}_{\mathbb{Q}}\left[\mathrm{e}^{\mathrm{i}\langle x, \ln F(t, \tau)\rangle} \mid \widetilde{F}_{t}\right] .
$$

The following pricing relation holds.

Proposition 4. Suppose that $g, \widehat{g} \in L^{1}\left(\mathbb{R}^{d}\right)$, where $g$ is defined in (10). Then

$$
C(t)=\mathrm{e}^{-r(T-t)} \frac{1}{2 \pi} \int_{\mathbb{R}^{d}} \widehat{g}(y) \exp \left(\widetilde{\phi}_{\ln F}^{(t, T, \tau)}(y)\right) \mathrm{d} y,
$$

where $\widetilde{\phi}_{\ln F}^{(t, T, \tau)}(y), t \leq T \leq \tau$, is the conditional characteristic function of $\ln F(T, \tau)$ defined in (11).

Proof. Since $g \in L^{1}\left(\mathbb{R}^{d}\right)$, using dominated convergence to commute integration and expectation (see $[10$, p. 54]), we conclude that

$$
\begin{aligned}
C(t) & =\mathrm{e}^{-r(T-t)} \mathbb{E}_{\mathbb{Q}}\left[f(F(T, \tau)) \mid \mathcal{F}_{t}\right] \\
& =\mathrm{e}^{-r(T-t)} \mathbb{E}_{\mathbb{Q}}\left[g(\ln F(T, \tau)) \mid \mathcal{F}_{t}\right] \\
& =\mathrm{e}^{-r(T-t)} \mathbb{E}_{\mathbb{Q}}\left[\frac{1}{2 \pi} \int_{\mathbb{R}^{d}} \widehat{g}(y) \mathrm{e}^{\mathrm{i}\langle y, \ln F(T, \tau)\rangle} \mathrm{d} y \mid \mathcal{F}_{t}\right] \\
& =\mathrm{e}^{-r(T-t)} \frac{1}{2 \pi} \int_{\mathbb{R}^{d}} \widehat{g}(y) \mathbb{E}_{\mathbb{Q}}\left[\mathrm{e}^{\mathrm{i}\langle y, \ln F(T, \tau)\rangle} \mid \widetilde{F}_{t}\right] \mathrm{d} y \\
& =\mathrm{e}^{-r(T-t)} \frac{1}{2 \pi} \int_{\mathbb{R}^{d}} \widehat{g}(y) \exp \left(\widetilde{\phi}_{\ln F}^{(t, T, \tau)}(y)\right) \mathrm{d} y .
\end{aligned}
$$

This completes the proof.

The two main ingredients in the pricing using Fourier methods are the transform of the payoff function, $\widehat{g}$, and the cumulant of the forward price under the pricing measure $\mathbb{Q}$. We state a semianalytical expression for the latter. 
Proposition 5. Assume that the conditions of Proposition 1 hold. Then the conditional cumulant function of $\ln F(t, \tau)$ for $s \leq t \leq \tau$ defined in (11) is

$$
\begin{aligned}
\widetilde{\phi}_{\ln F}^{(s, t, \tau)}(x)= & \mathrm{i} x^{\top} H(s, t, \tau)+\mathrm{i} x^{\top} \mathrm{e}^{A(\tau-s)} X(s)+\sum_{i=1}^{m} \mathrm{i} x^{\top} \mathrm{e}^{B_{i}(\tau-s)} Y_{i}(s) \\
& +\frac{1}{2} \sum_{j=1}^{n} \mathrm{i} x^{\top} \operatorname{diag}\left(D_{j}(t-s, \tau-t) Z_{j}(s)\right)-\frac{1}{2} \sum_{j=1}^{n} x^{\top} \mathcal{C}_{j}(\tau-s) Z_{j}(s) x \\
& +\Xi(s, t, \tau, x)
\end{aligned}
$$

for $x \in \mathbb{R}^{d}$, where

$$
\begin{aligned}
H(s, t, \tau)= & \ln \Lambda(\tau)+\ln \Psi(\tau-t)+A^{-1}\left(I-\mathrm{e}^{A(\tau-t)}\right) \theta_{0}+\sum_{i=1}^{m} B_{i}^{-1}\left(I-\mathrm{e}^{B_{i}(\tau-t)}\right) \mu_{i} \\
& +A^{-1}\left(\mathrm{e}^{A(\tau-t)}-\mathrm{e}^{A(\tau-s)}\right) \theta_{0}+\sum_{i=1}^{m} B_{i}^{-1}\left(\mathrm{e}^{B_{i}(\tau-t)}-\mathrm{e}^{B_{i}(\tau-s)}\right) \mu_{i}
\end{aligned}
$$

and

$$
\begin{gathered}
\Xi(s, t, \tau, x)=\sum_{j=1}^{m} \int_{0}^{t-s}\left\{\phi _ { \widetilde { L } _ { j } } \left(\frac{1}{2} \mathrm{i} \mathcal{C}_{j}^{*}(\tau-t+v)\left(x x^{\top}\right)+\frac{1}{2} \mathscr{D}_{j}^{*}(v, \tau-t)\left(J_{d}(x)\right)\right.\right. \\
\left.\left.\quad+J_{d}\left(x^{\top} \mathrm{e}^{B_{j}(\tau-t+v)} \eta_{j}\right)-\mathrm{i} \Theta_{j}\right)-\phi_{\widetilde{L}_{j}}\left(-\mathrm{i} \Theta_{j}\right)\right\} \mathrm{d} v \\
+\sum_{j=m+1}^{n} \int_{0}^{t-s}\left\{\phi_{\widetilde{L}_{j}}\left(\frac{1}{2} \mathrm{iC}_{j}^{*}(\tau-t+v)\left(x x^{\top}\right)+\frac{1}{2} \mathcal{D}_{j}^{*}(v, \tau-t)\left(J_{d}(x)\right)-\mathrm{i} \Theta_{j}\right)\right. \\
\left.-\phi_{\widetilde{L}_{j}}\left(-\mathrm{i} \Theta_{j}\right)\right\} \mathrm{d} v .
\end{gathered}
$$

The family of linear operators $\mathscr{D}_{j}(u, v),(u, v) \in \mathbb{R}_{+}^{2}$, are defined as

$$
\mathscr{D}_{j}(u, v) X=\mathcal{C}_{j}(v) \mathrm{e}^{C_{j} u} X \mathrm{e}^{C_{j}^{\top} u}
$$

for $j=1, \ldots, n$ and a matrix $X \in M_{d}(\mathbb{R})$.

Proof. From Proposition 1, it holds that

$$
\ln F(t, \tau)=\ln \Theta(t, \tau)+\mathrm{e}^{A(\tau)} X(t)+\sum_{i=1}^{m} \mathrm{e}^{B_{i}(\tau-t)} Y_{i}(t)+\frac{1}{2} \operatorname{diag}\left\{\sum_{j=1}^{n} \mathcal{C}_{j}(\tau-t) Z_{j}(t)\right\},
$$

where we recall the shorthand notation for $\Theta(t, \tau)$ defined in (9). Now, from the explicit solutions of the factors in (3), (4), and (5), together with the Girsanov change of the Brownian motion $W$, we find by adaptedness that

$$
\begin{aligned}
\widetilde{\phi}_{\ln F}^{(s, t, \tau)}(x)= & \mathrm{i} x^{\top} H(s, t, \tau)+\mathrm{i} x^{\top} \mathrm{e}^{A(\tau-s)} X(s)+\mathrm{i} x^{\top} \sum_{i=1}^{m} \mathrm{e}^{B_{i}(\tau-s)} Y_{i}(s) \\
& +\frac{1}{2} \mathrm{i} x^{\top} \operatorname{diag}\left\{\sum_{j=1}^{n} \mathscr{D}_{j}(t-s, \tau-t) Z_{j}(s)\right\}
\end{aligned}
$$




$$
\begin{aligned}
+\ln \mathbb{E}_{\mathbb{Q}}[ & \exp \left(\mathrm{i} x^{\top} \int_{s}^{t} \mathrm{e}^{A(\tau-u)} \Sigma^{1 / 2}(u) \mathrm{d} \widehat{W}(u)+\mathrm{i} x^{\top} \sum_{i=1}^{m} \int_{s}^{t} \mathrm{e}^{B_{i}(\tau-u)} \eta_{i} \mathrm{~d} L_{i}(u)\right. \\
+ & \left.\left.\frac{1}{2} \mathrm{i} x^{\top} \operatorname{diag}\left\{\sum_{j=1}^{n} \mathcal{C}_{j}(\tau-t) \int_{s}^{t} \mathrm{e}^{C_{j}(t-u)} \mathrm{d} \widetilde{L}_{j}(u) \mathrm{e}^{C_{j}^{\top}(t-u)}\right\}\right) \mid \mathcal{F}_{S}\right] .
\end{aligned}
$$

Define $\psi(s, t, \tau)$ as the logarithm of the conditional expectation in the expression above. Letting $g_{s, t}$ be the $\sigma$-algebra generated by $\mathcal{F}_{s}$ and the paths of $\widetilde{L}_{j}(u), s \leq u \leq t$, we find after using the tower property of the conditional expectation operator and the Gaussianity of Itô integrals of deterministic functions that

$$
\begin{aligned}
\psi(s, t, \tau)=\ln \mathbb{E}_{\mathbb{Q}}\left[\mathbb{E}_{\mathbb{Q}}\left[\exp \left(\mathrm{i} x^{\top} \int_{s}^{t} \mathrm{e}^{A(\tau-u)} \Sigma^{1 / 2}(u) \mathrm{d} \widehat{W}(u)\right) \mid g_{s, t} \widetilde{L}\right]\right. \\
\cdot \exp \left(\mathrm{i} x^{\top} \sum_{i=1}^{m} \int_{s}^{t} \mathrm{e}^{B_{i}(\tau-u)} \eta_{i} \mathrm{~d} L_{i}(u)\right) \\
\left.\cdot \exp \left(\frac{1}{2} \mathrm{i} x^{\top} \operatorname{diag}\left\{\sum_{j=1}^{n} \mathcal{C}_{j}(\tau-t) \int_{s}^{t} \mathrm{e}^{C_{j}(t-u)} \mathrm{d} \widetilde{L}_{j}(u) \mathrm{e}^{C_{j}^{\top}(t-u)}\right\}\right) \mid \mathcal{F}_{t}\right] \\
=\ln \mathbb{E}_{\mathbb{Q}}\left[\exp \left(-\frac{1}{2} x^{\top} \int_{s}^{t} \mathrm{e}^{A(\tau-u)} \Sigma(u) \mathrm{e}^{A^{\top}(\tau-u)} \mathrm{d} u x\right)\right. \\
\cdot \exp \left(\mathrm{i} x^{\top} \sum_{i=1}^{m} \int_{s}^{t} \mathrm{e}^{B_{i}(\tau-u)} \eta_{i} \mathrm{~d} L_{i}(u)\right) \\
\left.\cdot \exp \left(\frac{1}{2} \mathrm{i} x^{\top} \operatorname{diag}\left\{\sum_{j=1}^{n} \mathcal{C}_{j}(\tau-t) \int_{s}^{t} \mathrm{e}^{C_{j}(t-u)} \mathrm{d} \widetilde{L}_{j}(u) \mathrm{e}^{C_{j}^{\top}(t-u)}\right\}\right) \mid \mathcal{F}_{t}\right] .
\end{aligned}
$$

Inspecting the proof of Lemma 3, we find that

$$
\int_{s}^{t} \mathrm{e}^{A(\tau-u)} \Sigma(u) \mathrm{e}^{A^{\top}(\tau-u)} \mathrm{d} u=\sum_{j=1}^{n} \mathcal{C}_{j}(\tau-s) Z_{j}(s)+\int_{s}^{t} \mathcal{C}_{j}(\tau-u) \mathrm{d} \widetilde{L}_{j}(u) .
$$

By $\mathcal{F}_{s}$-adaptedness and the independent increment property of Lévy processes, it holds that

$$
\begin{aligned}
\psi(s, t, \tau)=- & \frac{1}{2} \sum_{j=1}^{n} x^{\top} \mathcal{C}_{j}(\tau-s) Z_{j}(s) x \\
+ & +\ln \mathbb{E}_{\mathbb{Q}}\left[\operatorname { e x p } \left(-\frac{1}{2} \sum_{j=1}^{n} x^{\top} \int_{s}^{t} \mathcal{C}_{j}(\tau-u) \mathrm{d} \widetilde{L}_{j}(u) x\right.\right. \\
& +\frac{1}{2} \mathrm{i} x^{\top} \operatorname{diag}\left\{\sum_{j=1}^{n} \mathcal{C}_{j}(\tau-t) \int_{s}^{t} \mathrm{e}^{C_{j}(t-u)} \mathrm{d} \widetilde{L}_{j}(u) \mathrm{e}^{C_{j}^{\top}(t-u)}\right\} \\
& \left.\left.+\sum_{i=1}^{m} \mathrm{i} x^{\top} \int_{s}^{t} \mathrm{e}^{B_{i}(\tau-u)} \eta_{i} \mathrm{~d} L_{i}(u)\right)\right]
\end{aligned}
$$


We focus next on the last term, the logarithm of the expectation, which we denote by $\widetilde{\psi}(s, t, \tau)$. Observe first that

$$
\mathcal{C}_{j}(\tau-t) \int_{s}^{t} \mathrm{e}^{C_{j}(t-u)} \mathrm{d} \widetilde{L}_{j}(u) \mathrm{e}^{C_{j}^{\top}(t-u)}=\int_{s}^{t} \mathscr{D}_{j}(t-u, \tau-t) \mathrm{d} \widetilde{L}_{j}(u) .
$$

But, since, for a matrix $A \in M_{d}(\mathbb{R}), x^{\top} \operatorname{diag}(A)=\operatorname{tr}\left\{J_{d}(x) A\right\}$,

$$
\frac{1}{2} x^{\top} \operatorname{diag}\left\{\sum_{j=1}^{n} \int_{s}^{t} \mathscr{D}_{j}(t-u, \tau-t) \mathrm{d} \widetilde{L}_{j}(u)\right\}=\sum_{j=1}^{n} \operatorname{tr}\left\{\frac{1}{2} J_{d}(x) \int_{s}^{t} \mathscr{D}_{j}(t-u, \tau-t) \mathrm{d} \widetilde{L}_{j}(u)\right\} .
$$

Furthermore, it holds that

$$
x^{\top} \int_{s}^{t} \mathrm{e}^{B_{i}(\tau-u)} \eta_{i} \mathrm{~d} L_{i}(u)=\int_{s}^{t} x^{\top} \mathrm{e}^{B_{i}(\tau-u)} \eta_{i} \mathrm{~d} L_{i}(u)=\operatorname{tr}\left\{\int_{s}^{t} J_{d}\left(x^{\top} \mathrm{e}^{B_{i}(\tau-u)} \mathrm{d} \widetilde{L}_{i}(u)\right\}\right.
$$

and

$$
-\frac{1}{2} x^{\top} \int_{s}^{t} \mathcal{C}_{j}(\tau-u) \mathrm{d} \widetilde{L}_{j}(u) x=\operatorname{itr}\left\{\frac{1}{2} \mathrm{i} x x^{\top} \int_{s}^{t} \mathcal{C}_{j}(\tau-u) \mathrm{d} \widetilde{L}_{j}(u)\right\} .
$$

Hence, collecting terms and using the fact that the $\widetilde{L}_{j}$ are independent for $j=1, \ldots, n$, we find that

$$
\begin{aligned}
& \widetilde{\psi}(s, t, \tau)=\sum_{j=1}^{m} \ln \mathbb{E}_{\mathbb{Q}}\left[\operatorname { e x p } \left(\operatorname { i t r } \left\{\frac{1}{2} \mathrm{i} x x^{\top} \int_{s}^{t} \mathcal{C}_{j}(\tau-u) \mathrm{d} \widetilde{L}_{j}(u)\right.\right.\right. \\
& +\frac{1}{2} J_{d}(x) \int_{s}^{t} \mathscr{D}_{j}(t-u, \tau-t) \mathrm{d} \tilde{L}_{j}(u) \\
& \left.\left.\left.+\int_{s}^{t} J_{d}\left(x^{\top} \mathrm{e}^{B_{j}(\tau-u)} \eta_{j}\right) \mathrm{d} \tilde{L}_{j}(u)\right\}\right)\right] \\
& +\sum_{j=m+1}^{n} \ln \mathbb{E}_{\mathbb{Q}}\left[\operatorname { e x p } \left(\operatorname { i t r } \left\{\frac{1}{2} \mathrm{i} x x^{\top} \int_{s}^{t} \mathcal{C}_{j}(\tau-u) \mathrm{d} \widetilde{L}_{j}(u)\right.\right.\right. \\
& \left.\left.\left.+\frac{1}{2} J_{d}(x) \int_{s}^{t} \mathscr{D}_{j}(t-u, \tau-t) \mathrm{d} \widetilde{L}_{j}(u)\right\}\right)\right] \\
& =\sum_{j=1}^{m} \int_{s}^{t}\left\{\phi _ { \widetilde { L } _ { j } } \left(\frac{1}{2} \mathrm{i} \mathcal{C}_{j}^{*}(\tau-u)\left(x x^{\top}\right)+\frac{1}{2} \mathscr{D}_{j}^{*}(t-u, \tau-t)\left(J_{d}(x)\right)\right.\right. \\
& \left.\left.+J_{d}\left(x^{\top} \mathrm{e}^{B_{j}(\tau-u)} \eta_{j}\right)-\mathrm{i} \Theta_{j}\right)-\phi_{\widetilde{L}_{j}}\left(-\mathrm{i} \Theta_{j}\right)\right\} \mathrm{d} u \\
& +\sum_{j=m+1}^{n} \int_{s}^{t}\left\{\phi_{\widetilde{L}_{j}}\left(\frac{1}{2} \mathrm{i} \mathcal{C}_{j}^{*}(\tau-u)\left(x x^{\top}\right)+\frac{1}{2} \mathscr{D}_{j}^{*}(t-u, \tau-t)\left(J_{d}(x)\right)-\mathrm{i} \Theta_{j}\right)\right. \\
& \left.-\phi_{\widetilde{L}_{j}}\left(-\mathrm{i} \Theta_{j}\right)\right\} \mathrm{d} u \text {. }
\end{aligned}
$$

In the last equality, we used the same argument as used in the proof of Proposition 1. After collecting terms, the proposition is proved. 
The fast Fourier transform (FFT) algorithm may be used to compute the option price efficiently, as long as we know the Fourier transform of the payoff function $g$. Note that implementing the FFT algorithm requires some numerical integration routines to evaluate the characteristic function of $\ln F$.

We consider the specific case of a call option on the spread between two forwards. The payoff function of such a contract is $f(x)=\max \left(x_{1}-x_{2}-K, 0\right)$, where $K$ is the strike price. Without loss of generality, we can suppose that $K=1$. The function $g$ becomes

$$
g(x)=\max \left(\mathrm{e}^{x_{1}}-\mathrm{e}^{x_{2}}-1,0\right) .
$$

We observe that this function is not integrable on $\mathbb{R}^{2}$. However, following the idea in [8], we can dampen $g$ by an exponential function. To this end, define, for $\xi=\left(\xi_{1},-\xi_{2}\right)$ with $\xi_{1}, \xi_{2}>0$,

$$
g_{\xi}(x)=\mathrm{e}^{-\langle\xi, x\rangle} \max \left(\mathrm{e}^{x_{1}}-\mathrm{e}^{x_{2}}-1,0\right)
$$

We show that this becomes an integrable function under natural conditions on the damping factors $\xi_{1}$ and $\xi_{2}$.

Lemma 4. If $\xi_{1}-\xi_{2}>1$ then $g_{\xi} \in L^{1}\left(\mathbb{R}^{2}\right)$, where $g_{\xi}$ is defined in (12).

Proof. Note that the function $g_{\xi}$ is nonzero whenever $x_{1}>\ln \left(\mathrm{e}^{x_{2}}+1\right)$. Thus, since $\xi_{1}>1$,

$$
\begin{aligned}
\int_{\mathbb{R}^{2}} g_{\xi}(x) \mathrm{d} x & =\int_{-\infty}^{\infty} \mathrm{e}^{\xi_{2} x_{2}} \int_{\ln \left(\mathrm{e}^{x_{2}}+1\right)}^{\infty} \mathrm{e}^{-\xi_{1} x_{1}}\left(\mathrm{e}^{x_{1}}-\left(\mathrm{e}^{x_{2}}+1\right)\right) \mathrm{d} x_{1} \mathrm{~d} x_{2} \\
& =\frac{1}{\xi_{1}\left(\xi_{1}-1\right)} \int_{-\infty}^{\infty} \mathrm{e}^{\xi_{2} x_{2}}\left(\mathrm{e}^{x_{2}}+1\right)^{-\left(\xi_{1}-1\right)} \mathrm{d} x_{2} .
\end{aligned}
$$

If $x_{2}>0$, we find that

$$
\mathrm{e}^{\xi_{2} x_{2}}\left(\mathrm{e}^{x_{2}}+1\right)^{-\left(\xi_{1}-1\right)}=\mathrm{e}^{\xi_{2} x_{2}} \mathrm{e}^{-\left(\xi_{1}-1\right) x_{2}}\left(1+\mathrm{e}^{-x_{2}}\right)^{-\left(\xi_{1}-1\right)} \leq \mathrm{e}^{\left(\xi_{2}-\xi_{1}+1\right) x_{2}} .
$$

By assumption on $\xi_{1}$ and $\xi_{2}$, we have $\xi_{2}-\xi_{1}+1<0$. If $x_{2}<0$ then

$$
\mathrm{e}^{\xi_{2} x_{2}}\left(\mathrm{e}^{x_{2}}+1\right)^{-\left(\xi_{1}-1\right)} \leq \mathrm{e}^{\xi_{2} x_{2}}
$$

This completes the proof.

In the next lemma we state the Fourier transform of $g_{\xi}$.

Lemma 5. Suppose that $\xi_{2}>0$ and $\xi_{1}-\xi_{2}>1$. Then the Fourier transform of $g_{\xi}(x)$ defined in (12) is

$$
\widehat{g}_{\xi}(y)=\frac{\Gamma\left(\mathrm{i}\left(y_{1}+y_{2}\right)-\left(1+\xi_{1}+\xi_{2}\right)\right) \Gamma\left(-\mathrm{i} y_{2}+\xi_{2}+2\right)}{\Gamma\left(\mathrm{i} y_{1}+1-\xi_{1}\right)},
$$

where $\Gamma$ denotes the gamma function.

Proof. For the proof, we follow the approach of [13, Theorem 1]. When one takes into account the exponential damping of the pay-off function $g$ by $\mathrm{e}^{\langle\xi, x\rangle}$ then the above result follows.

We have

$$
g(x)=\frac{1}{2 \pi} \int_{\mathbb{R}^{2}} \widehat{g}_{\xi}(y) \mathrm{e}^{\mathrm{i}\langle(y-\mathrm{i} \xi), x\rangle} \mathrm{d} y .
$$


Thus, the price of a spread option entails substituting $y$ with $y-\mathrm{i} \xi$ in the formula for $C(t)$ in Proposition 4 , using $\widehat{g}_{\xi}$ instead of $\widehat{g}$. In addition, we require an exponential integrability condition on $\ln F(T, \tau)$ in order to take into account the additional contribution from $\exp (\langle\xi, x\rangle)$.

An alternative approach to the Fourier method is to apply Monte Carlo simulation of the forward price dynamics $F(T, \tau)$ in the pricing of options. In practice, this means simulating matrix-valued subordinators $\widetilde{L}_{j}$ and a multidimensional Wiener processes $\widehat{W}$ under $\mathbb{Q}$. The latter can be simulated using classical sampling techniques. Finding efficient simulation methods for matrix-valued subordinators is in general an open problem; however, for a specific class of such processes a method is proposed by Benth and Vos [6].

\section{Conclusions}

Based on the multivariate spot price model with Barndorff-Nielsen and Shephard stochastic volatility introduced in Benth and Vos [6], we derived the multivariate forward price dynamics. These analytical forward prices were calculated based on a combined Esscher-Girsanov change of measure where the risk premium is parametrized into a spike and volatility premium. Although the spot price has continuous sample paths in absence of a spike process, the implied forward curve will still exhibit jumps inherited from the stochastic volatility process. In the long end of the market the forward prices are basically equal to the seasonality function adjusted by the long-term means of the spike processes and volatility process and the market prices of risk. Since the mean-reverting structure of the involved matrix exponentials have a richer structure than in the one-dimensional case, the implied forward curve can alternate between backwardation and contango and humps may appear. Depending on the time to maturity a change in the spot can lead to various changes in the forward curve. We also discussed how a transform-based method can be used in order to price cross-commodity options on forwards. The particular case of spread options were analyzed in more detail.

\section{Acknowledgement}

Fred Espen Benth acknowledges the financial support from the project 'Energy markets: modelling, optimization and simulation' (EMMOS), funded by the Norwegian Research Council through the eVITA grant 205328.

\section{References}

[1] Andresen, A., Benth, F. E., Koekebakker, S. and Zakamouline, V. (2011). The CARMA interest rate model. In preparation.

[2] BarndorfF-Nielsen, O. E. And Shephard, N. (2001). Non-Gaussian Ornstein-Uhlenbeck-based models and some of their uses in economics. J. R. Statist. Soc. B 63, 167-241.

[3] Barndorff-Nielsen, O. E. ANd Stelzer, R. (2007). Positive-definite matrix processes of finite variation. Prob. Math. Statist. 27, 3-43.

[4] Barndorff-Nielsen, O. E., Pedersen, J. and Sato, K.-I. (2001). Multivariate subordination, self-decomposability and stability. Adv. Appl. Prob. 33, 160-187.

[5] BENTH, F. E. (2011). The stochastic volatility model of Barndorff-Nielsen and Shephard in commodity markets. Math. Finance 21, 595-625.

[6] Benth, F. E. AND Vos, L. (2013). Cross-commodity spot price modeling with stochastic volatility and leverage for energy markets. Adv. Appl. Prob. 45, 545-571.

[7] Benth, F. E., Š Saltytė Benth, J. And KoeKebaKker, S. (2008). Stochastic Modelling of Electricity and Related Markets. World Scientific, Hackensack, NJ.

[8] Carr, P. And Madan, D. B. (1999). Option valuation using the fast Fourier transform. J. Comput. Finance 2, 61-73.

[9] Duffie, D. (1992). Dynamic Asset Pricing Theory. Princeton University Press, Princeton. 
[10] Folland, G. B. (1984). Real Analysis. John Wiley, New York.

[11] Geman, H. (2005). Commodities and Commodity Derivatives. John Wiley, Chichester.

[12] Horn, R. A. And Johnson, C. R. (1985). Matrix analysis. Cambridge University Press.

[13] Hurd, T. R. ANd Zhou, Z. (2009). A Fourier transform method for spread option pricing. SIAM J. Financial Math. 1, 142-157.

[14] IKeda, N. And Watanabe, S. (1981). Stochastic Differential Equations and Diffusion Processes. North-Holland, Amsterdam.

[15] Karatzas, I. and Shreve, S. E. (1991). Brownian Motion and Stochastic Calculus. Springer, New York.

[16] Pigorsch, C. And Stelzer, R. (2009). A multivariate Ornstein-Uhlenbeck type stochastic volatility model. Eprint. Available at http://www-m4.ma.tum.de.

[17] Protter, P. (1990). Stochastic Integration and Differential Equations. Springer, Berlin.

[18] Schwartz, E. S. (1997). The stochastic behavior of commodity prices: implications for valuation and hedging. J. Finance 52, 923-973.

[19] Shiryaev, A. N. (1999). Essentials of Stochastic Finance. World Scientific, River Edge, NJ.

[20] Trolle, A. B. and Schwartz, E. S. (2009). Unspanned stochastic volatility and the pricing of commodity derivatives. Rev. Financial Studies 22, 4423-4461. 